\title{
DNS of vertical plane channel flow with finite-size particles: Voronoi analysis, acceleration statistics and particle-conditioned averaging
}

\author{
Manuel García-Villalba ${ }^{a}$, Aman G. Kidanemariam $^{b}$ and Markus Uhlmann ${ }^{b} *$ \\ a Bioingeniería e Ingeniería Aeroespacial, Universidad Carlos III de Madrid, Leganés 28911, Spain \\ ${ }^{b}$ Institute for Hydromechanics, Karlsruhe Institute of Technology (KIT), 76131 Karlsruhe, Germany
}

\begin{abstract}
We have performed a direct numerical simulation of dilute turbulent particulate flow in a vertical plane channel, fully resolving the phase interfaces. The flow conditions are the same as those in the main case of "Uhlmann, M., Phys. Fluids, vol. 20, 2008, 053305", with the exception of the computational domain length which has been doubled in the present study. The statistics of flow and particle motion are not significantly altered by the elongation of the domain. The large-scale columnar-like structures which had previously been identified do persist and they are still only marginally decorrelated in the prolonged domain. Voronoi analysis of the spatial particle distribution shows that the state of the dispersed phase can be characterized as slightly more ordered than random tending towards a homogeneous spatial distribution. It is also found that the p.d.f.'s of Lagrangian particle accelerations for wall-normal and spanwise directions follow a lognormal distribution as observed in previous experiments of homogeneous flows. The streamwise component deviates from this law presenting significant skewness. Finally, a statistical analysis of the flow in the near field around the particles reveals that particle wakes present two regions, a near wake where the velocity deficit decays as $x^{-1}$ and a far wake with a decay of approximately $x^{-2}$.
\end{abstract}

Keywords: particulate flow, direct numerical simulation, interface resolution, turbulent channel flow

\section{Introduction}

Fluid flow with suspended solid particles is encountered in a multitude of natural and industrial systems. Examples include the motion of sediment particles in rivers, fluidized beds and blood flow. Despite the great technological importance of these systems our understanding of the dynamics of fluid-particle interaction is still incomplete at the present date. Recently, however, significant progress has been made based on data provided by new experimental methods as well as numerical simulations. While most past investigations of numerical type have been performed in the context of the point-particle approach, it has now become possible to simulate the motion of a considerable number of finite-size particles including an accurate description of the surrounding flow field on the particle scale (Pan and Banerjee, 1997; Kajishima and Takiguchi, 2002; Ten Cate et al., 2004; Uhlmann, 2008; Lucci, Ferrante and Elghobashi, 2010, 2011). Although the complexity of these particle-resolved simulations (in terms of Reynolds number, number of particles and computational domain size) is still limited, new insight into the physics of fluid-particle systems is beginning to emerge from such studies.

Uhlmann (2008) has simulated turbulent flow in a vertically-oriented plane channel seeded with heavy spherical particles with a diameter corresponding to approximately 11 wall units at a

*Corresponding author: markus.uhlmann@kit.edu 
solid volume fraction of $0.4 \%$. The pressure-driven upward flow (at constant flow rate) was found to be strongly modified due to the particle presence, with increased wall-shear stress and strongly enhanced turbulence intensity. The average relative flow, corresponding to a Reynolds number (based on particle diameter) of approximately 135, lead to the establishment of wakes behind individual particles. Additionally, the formation of very large-scale, streak-like flow structures (essentially spanning the entire box-size), absent in corresponding single-phase flow, was observed. At the same time the dispersed phase did not exhibit any of the common signs of preferential concentration.

In the present study we are revisiting the same flow configuration of vertical particulate channel flow, expanding upon the previous analysis of Uhlmann (2008) by addressing several unanswered questions. First, we wish to determine the influence of the streamwise length of the computational domain upon the largest flow scales. For this purpose we have performed new simulations analogous to the ones conducted by Uhlmann (2008), but with twice the value of the original streamwise period, while keeping all remaining parameters unchanged.

Second, we intend to provide a more complete description of the turbulent fluid-particle interaction in vertical channel flow. To this end we have analyzed three aspects of the flow dynamics which had previously not been considered by Uhlmann (2008): Voronoi analysis of the spatial structure of the dispersed phase, analysis of particle acceleration statistics, and particle-conditioned averaging of the fluid flow field.

Voronoi analysis is a relatively recent addition to the arsenal of tools for the description of particles suspended in fluids (Monchaux, Bourgoin and Cartellier, 2010). In the present flow configuration it turns out that this methodology provides a more sensitive measure of the particle phase geometry than previously employed criteria.

The statistical properties of particle acceleration have received increasing attention in recent years (Toschi and Bodenschatz, 2009). Since particle acceleration is (up to particle mass) equivalent to the resulting forces acting upon the particles, its analysis can be instrumental in understanding turbulence-particle interaction mechanisms. One application where the influence of turbulence upon particle acceleration statistics is believed to be of key importance is the growth of rain drops by collisions in atmospheric clouds (Warhaft, 2009). Modern experimental results on the acceleration of finite-size particles (Qureshi et al., 2007; Xu and Bodenschatz, 2008; Brown, Warhaft and Voth, 2009) have only started to emerge around the date of publication of the precursor paper (Uhlmann, 2008). Therefore, such an analysis was not carried out therein. Here we present a statistical analysis of particle acceleration/hydrodynamic forces, relating the findings to available experimental results.

Finally, the understanding of the interaction between solid particles and fluid turbulence does not seem complete without a statistical analysis of the flow in the near-field around the particles. In order to investigate the characteristics of particle-induced wakes and with the aim to provide data which might be useful for the purpose of two-phase flow modelling, we have undertaken a study of particle-conditioned averaging of the flow field. Reference data for fixed particles swept by (essentially) homogeneous-isotropic flow (Bagchi and Balachandar, 2004; Amoura et al., 2010) as well as wall-bounded shear flow (Wu and Faeth, 1994; Legendre, Merle and Magnaudet, 2006; Zeng, Balachandar and Najjar, 2010) is available and has been used for the purpose of comparison.

\section{Computational setup}

\subsection{Numerical method}

The numerical method employed in the current simulations is identical to the one detailed in Uhlmann (2005) which was already used for the previous simulations of vertical particulate channel flow by Uhlmann (2008). The incompressible Navier-Stokes equations are solved by a fractional step approach with implicit treatment of the viscous terms (Crank-Nicolson) and a three-step Runge-Kutta scheme for the non-linear terms. The spatial discretization employs second-order central finite-differences on a staggered mesh. The no-slip condition at the surface of moving solid 
(a)

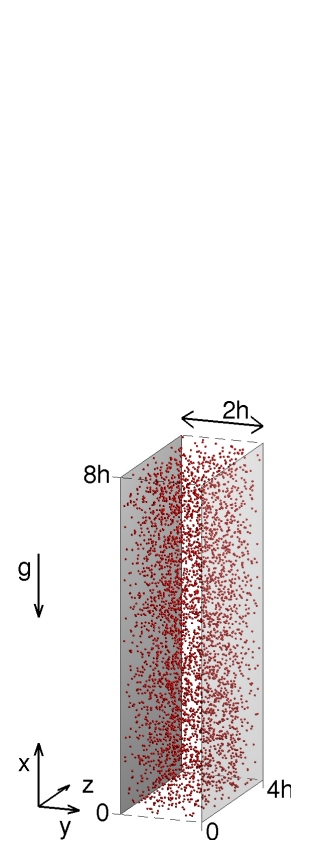

(b)

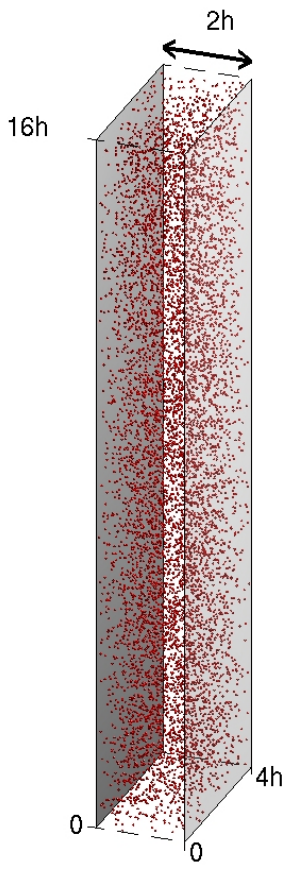

Figure 1: Illustration of the computational domain, which is bi-periodic in the streamwise $(x)$ and spanwise $(z)$ directions. (a) shows the domain used in Uhlmann (2008), (b) the current domain which is a streamwise extension of the former (by a factor of two). The red spheres indicate actual instantaneous particle positions.

$$
\begin{array}{ccccccccc}
R e_{b} & R e_{\tau} & \frac{\rho_{p}}{\rho_{f}} & |\mathbf{g}| h / u_{b}^{2} & S t^{+} & S t_{b} & h / D & D^{+} & \Phi_{s} \\
2700 & 220.9 & 2.2077 & 12.108 & 15.5 & 0.83 & 20 & 11.25 & 0.0042
\end{array}
$$

Table 1: Physical parameters for particulate flow in a vertically oriented plane channel: bulk Reynolds number $R e_{b}$ (imposed quantity), friction-velocity based Reynolds number $R e_{\tau}$ (derived quantity), particle/fluid density ratio $\rho_{p} / \rho_{f}$, gravitational parameter $|\mathbf{g}| h / u_{b}^{2}$, Stokes numbers based upon bulk units $S t_{b}=\tau_{p} u_{b} / h$ (imposed) and wall units $S t^{+}=\tau_{p} u_{\tau}^{2} / \nu$ (derived), length scale ratio $h / D$ (imposed) and $D^{+}$(derived), and global solid volume fraction $\Phi_{s}$. The particle relaxation time scale was defined as $\tau_{p}=\rho_{p} D^{2} /\left(\rho_{f} 18 \nu\right)$.

particles is imposed by means of a specially designed immersed boundary technique (Uhlmann, 2005). The motion of the particles is computed from the Newton equations for linear and angular motion of rigid bodies, driven by buoyancy, hydrodynamic forces/torque and contact forces (in case of collisions). Since the suspension under consideration is dilute, collisions are treated by a simple repulsive force mechanism (Glowinski et al., 1999) formulated such as to keep colliding particles from overlapping non-physically. The same treatment is applied to particle-wall encounters. It should be noted that the employed computational grid is uniform and isotropic. The chosen grid width $\Delta x=\Delta y=\Delta z$ yields a particle resolution of $D / \Delta x=12.8$, a resolution of the channel half-width of $h / \Delta x=256$ and $\Delta x^{+}=0.67$ in terms of wall units. Further information on our extensive validation tests and grid convergence can be found in Uhlmann $(2005,2008)$ and further references therein. 


$\begin{array}{ccccc}\text { case } & \Omega & N_{x} \times N_{y} \times N_{z} & N_{p} & t_{o b s} u_{b} / h \\ \text { Uhlmann (2008) } & 8 h \times 2 h \times 4 h & 2048 \times 513 \times 1024 & 4096 & 115 \\ \text { present } & 16 h \times 2 h \times 4 h & 4096 \times 513 \times 1024 & 8192 & 90\end{array}$

Table 2: Numerical parameters employed in the simulations: computational domain size $\Omega$, number of grid nodes $N_{i}$ in the $i$ th coordinate direction, number of particles $N_{p}$, temporal observation interval $t_{o b s}$ after discarding the initial transient. The grid spacing in all cases is fixed at $\Delta x=$ $h / 256$, corresponding to $N_{L}=515$ Lagrangian force points distributed over the surface of each particle.

$(a)$

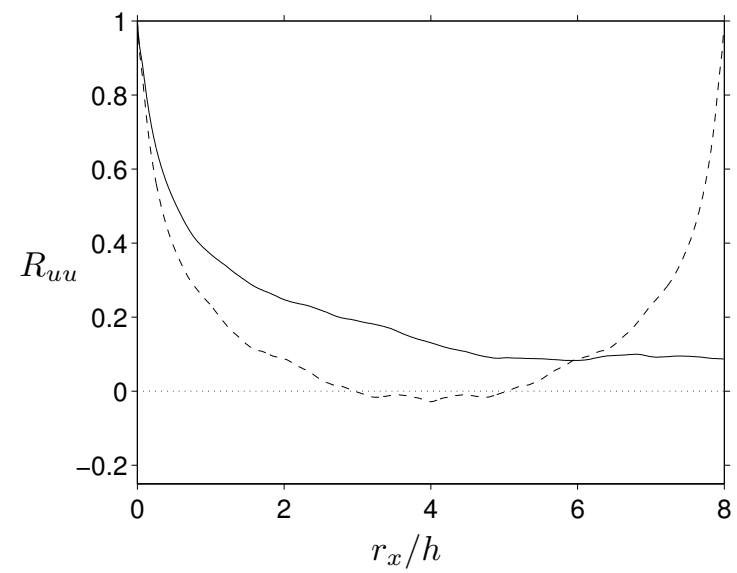

$(b)$

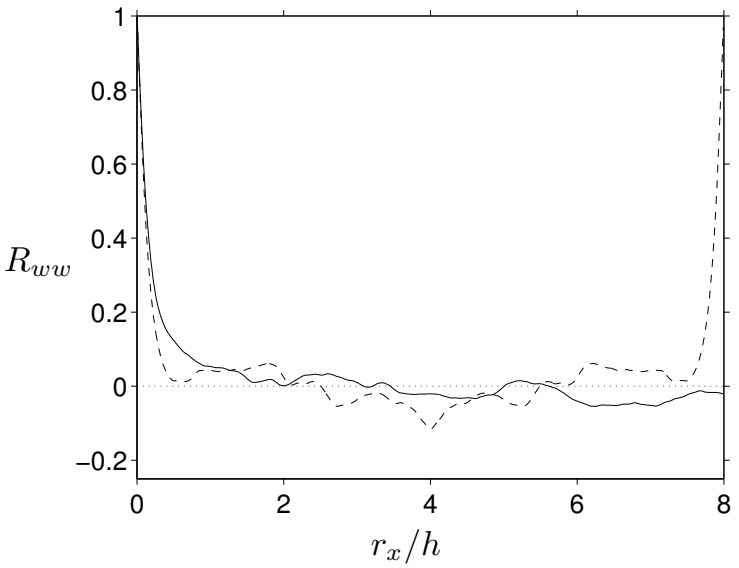

Figure 2: Two-point correlation functions of fluid phase velocity fluctuations for streamwise separations computed from a single snapshot of the flow field in a wall-parallel plane at a wall distance of $y / h=0.0938$ (i.e. $y^{+} \approx 22$ ). (a) streamwise velocity component $R_{u u}$, (b) spanwise velocity component $R_{w w}$. The correlation immediately after periodic extension from the small-box simulation of Uhlmann (2008) is shown as a dashed line; the correlation at the beginning of the present sampling interval is indicated by a solid line.

\subsection{Flow configuration}

Figure 1 shows the flow configuration under consideration as well as the coordinate system. The plane channel is oriented vertically, $x$ being the streamwise coordinate direction, $y$ is the wallnormal (with the channel width equal to $2 h$ ) and $z$ the spanwise direction. Fluid flow is directed upwards (in the positive $x$ direction), driven by a streamwise pressure-gradient. The bulk velocity $u_{b}$ is maintained at a constant value, such that the Reynolds number based upon the bulk velocity, $R e_{b}=u_{b} h / \nu$, is imposed (cf. table 1 for the values of the principal physical parameters). A large number $\left(N_{p}=8192\right)$ of monodispersed, rigid, spherical particles is suspended in the flow. The nominal terminal velocity of the particles (computed from an equilibrium of buoyancy force and standard drag force, Clift, Grace and Weber, 1978) is set equal to the bulk velocity of the fluid phase. Consequently, the average particle settling velocity obtained in the actual simulation is roughly zero. The chosen density ratio $\rho_{p} / \rho_{f}=2.2077$ (where $\rho_{p}, \rho_{f}$ are the particle and fluid densities) is comparable to the case of glass particles in water. The particle diameter $D$, approximately equal to 11 wall units, is comparable to the cross-sectional scales of buffer layer flow structures. Finally, table 1 shows that the suspension is indeed dilute, with less than one half percent of solid volume fraction.

As can be seen from table 2 , the present simulation is performed in a computational domain which has twice the streamwise period as compared to Uhlmann (2008), while maintaining an identical small-scale resolution. The table also shows that an observation interval of approximately 90 bulk time units (defined as $T_{b}=h / u_{b}$ ) has been computed after discarding the initial transient, 
(a)

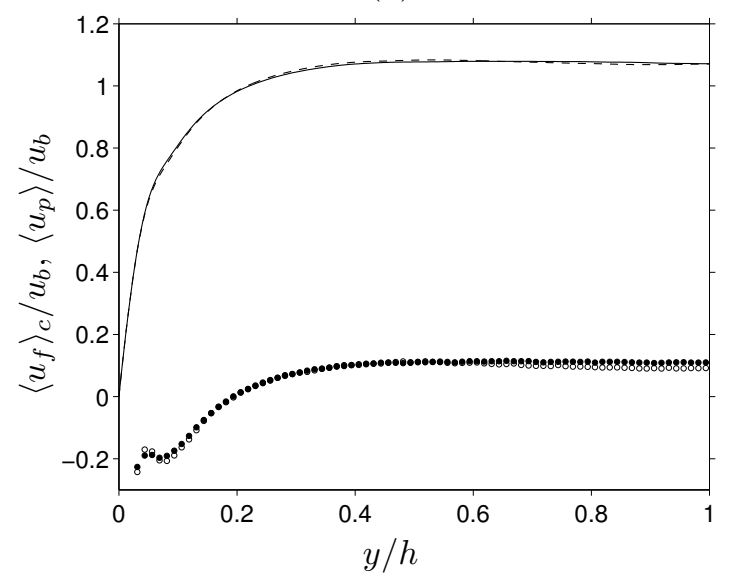

(b)

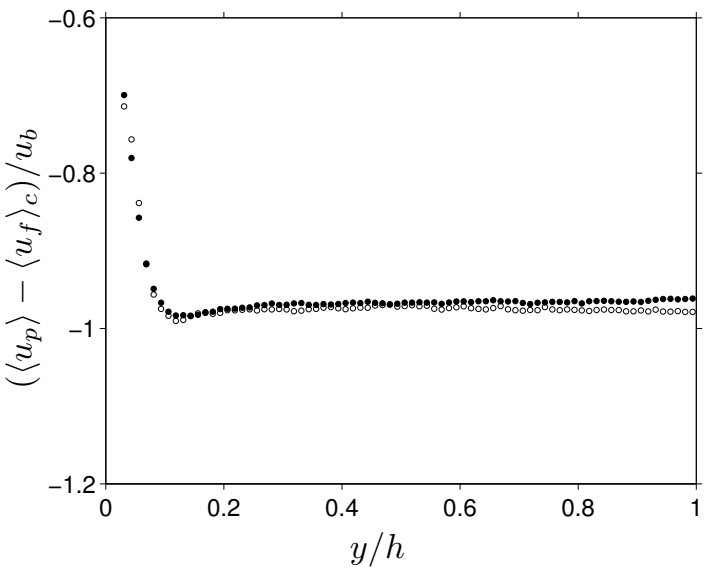

Figure 3: (a) Mean velocity profiles of both phases. The fluid phase is shown as: ——, present; ----, Uhlmann (2008). The mean velocity of the particulate phase is shown as: •, present; $\circ$, Uhlmann (2008). (b) Apparent slip velocity between the phases: •, present; o, Uhlmann (2008). Fluid data shown in this figure is 'composite-averaged' (cf. equation 23) for the purpose of strict comparison; pure fluid averaging data for the present simulation is shown in Appendix B.

which will be discussed next.

The simulations were run on different supercomputing systems, typically using between 256 and 1024 processor cores. The total number of CPU hours spent was of the order of 5 million.

\subsection{Simulation start-up and initial transient}

The current simulation was initialized with an exact periodic extension of a flow field taken at an instant towards the end of the simulation of Uhlmann (2008). For this purpose, fluid and particle data in the interval $x \in[0,8 h]$ was copied from the reference field. In order to obtain data in the interval $x \in[8 h, 16 h]$, the shift $x=\tilde{x}+8 h$ was applied to the reference field. It should be emphasized that no explicit perturbations whatsoever were added to the initial field.

Subsequently, the extended simulation was run while different quantities were monitored in order to determine whether the system has developed sufficiently such as to "forget" the initial state. A sensitive measure of independence from the initial condition is provided by two-point correlations of fluid data. Therefore, correlation functions of fluid velocity components as a function of streamwise separations have been analyzed. As can be seen from figure 2, the field initially exhibits a periodicity over half the domain length, consistent with the fact that the simulation was started from an exact "copy" of a field taken from a run in a domain with half the streamwise period. Over an interval of approximately $40 T_{b}$ the artificial periodicity (with period $8 h$ ) gradually disappears and only the strict periodicity over the fundamental period of $16 h$ remains. The transient interval was discarded and statistics have then been accumulated over a sampling interval of $90 T_{b}$. The plots in figure 2 also show the correlation function computed from a snapshot at the beginning of the interval over which statistics have been accumulated, which clearly lacks any traces of artificial periodicity.

\subsection{Notation}

Before turning to the results, let us fix the basic notation followed throughout the present text. Velocity vectors and their components corresponding to the fluid and the particle phases are distinguished by subscripts " $\mathrm{f}$ " and "p", respectively, as in $\mathbf{u}_{f}=\left(u_{f}, v_{f}, w_{f}\right)^{T}$ and $\mathbf{u}_{p}=\left(u_{p}, v_{p}, w_{p}\right)^{T}$. Similarly, the vector of angular particle velocity is denoted as $\boldsymbol{\omega}_{p}=\left(\omega_{p 1}, \omega_{p 2}, \omega_{p 3}\right)^{T}$ and linear particle acceleration as $\mathbf{a}_{p}=\left(a_{p 1}, a_{p 2}, a_{p 3}\right)^{T}$, while fluid acceleration is denoted as $\mathbf{a}_{f}=$ 
(a)

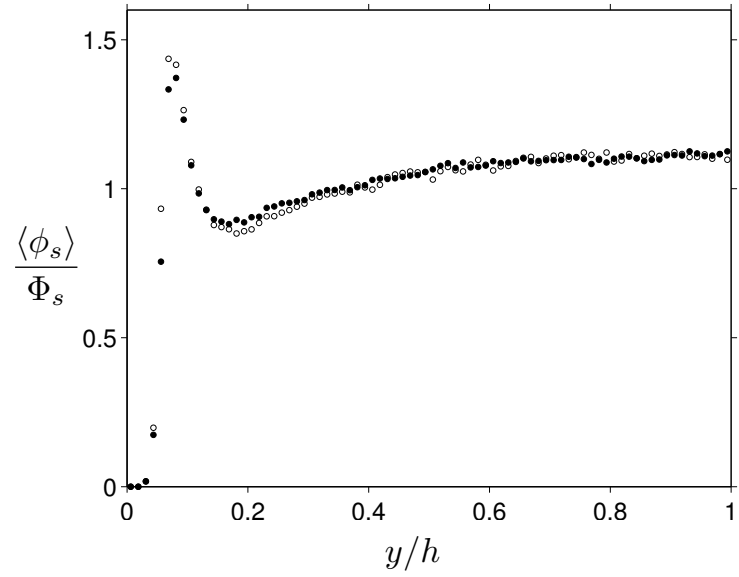

(b)

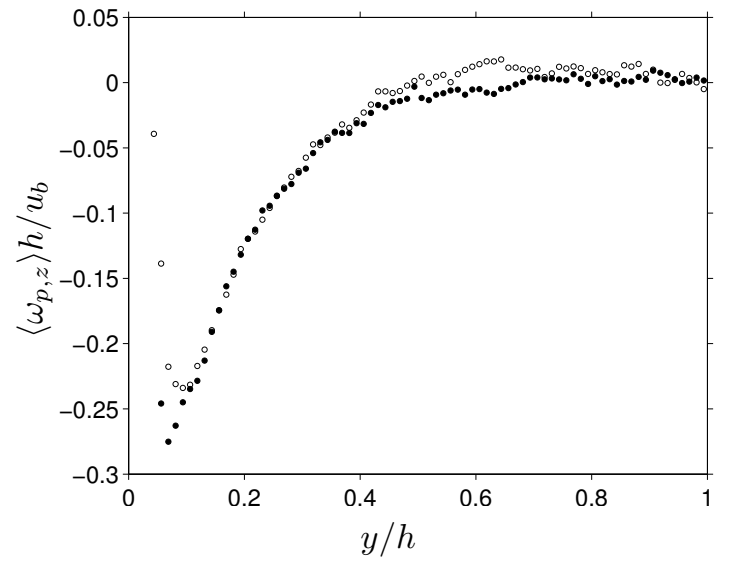

Figure 4: Wall normal profiles of: $(a)$ the mean solid volume fraction, $(b)$ the mean value of the spanwise component of angular particle velocity. Symbols as in figure $3(b)$.

$\left(a_{f 1}, a_{f 2}, a_{f 3}\right)^{T}$. The equations of motion for an immersed sphere with density $\rho_{p}$ can be written as:

$$
\begin{aligned}
\rho_{p} V_{p} \mathbf{a}_{p} & =\mathbf{F}_{H}+\mathbf{F}_{B}+\mathbf{F}_{C}, \\
I_{p} \dot{\boldsymbol{\omega}}_{p} & =\mathbf{T}_{H},
\end{aligned}
$$

where $V_{p}=\pi D^{3} / 6$ is the volume of a sphere with diameter $D$ and $I_{p}=\rho_{p} D^{5} \pi / 60$ is its moment of inertia, considering a homogeneous mass density. In equation (1a) the resulting force has been separated into a hydrodynamic contribution $\mathbf{F}_{H}=\int_{\mathcal{S}} \boldsymbol{\tau} \cdot \mathbf{n} \mathrm{d} \sigma-\int_{\mathcal{S}} p \mathbf{n} \mathrm{d} \sigma$ (S being the surface of the sphere, $\mathbf{n}$ the outward pointing normal vector at the surface, $\boldsymbol{\tau}=\rho_{f} \nu\left(\partial u_{f i} / \partial x_{j}+\partial u_{f j} / \partial x_{i}\right)$ the viscous stress tensor and $p$ the hydrodynamic pressure), a relative buoyancy force $\mathbf{F}_{B}=$ $\left(\rho_{p}-\rho_{f}\right) \mathbf{g} V_{p}$ and a contribution $\mathbf{F}_{C}$ from solid-solid contact which is modelled as discussed in $\S 2.1$. The angular acceleration in (1b) only has a single contribution from viscous hydrodynamic stresses, viz. $\mathbf{T}_{H}=\int_{\mathcal{S}} \mathbf{r}_{c} \times(\boldsymbol{\tau} \cdot \mathbf{n}) \mathrm{d} \sigma$ (r. $\mathbf{r}_{c}$ being the distance vector from the particle center), since our solid-solid collision treatment is limited to normal forces.

\section{Results}

\subsection{Effect of streamwise elongated domain}

The aim of the present section is to examine the effect of the finite streamwise period imposed upon the fields in the simulation. For this purpose we will compare the present results with those obtained in a shorter domain as presented by Uhlmann (2008).

Note that the fluid data shown in figures 3 and 5 are based upon 'composite' averaging, i.e. not distinguishing between velocity values of those grid nodes which are instantaneously located in the fluid phase and those inside the solid phase. Doing so allows for a strict comparison with results from the simulation of Uhlmann (2008), where this distinction was not made (cf. discussion in Appendix A of Uhlmann, 2008). The specific fluid-phase statistics (including only actual grid nodes located inside the fluid domain, cf. definition in equation 22) corresponding to the fluid quantities shown in figures 3 and 5 are included in Appendix B for future reference.

In the present flow it turns out that the average time interval between two collision events experienced by a particle is $3.9 T_{b}$, showing that indeed collisions are relatively infrequent. Furthermore, no particle-wall collisions have been observed during the simulation interval. 
(a)

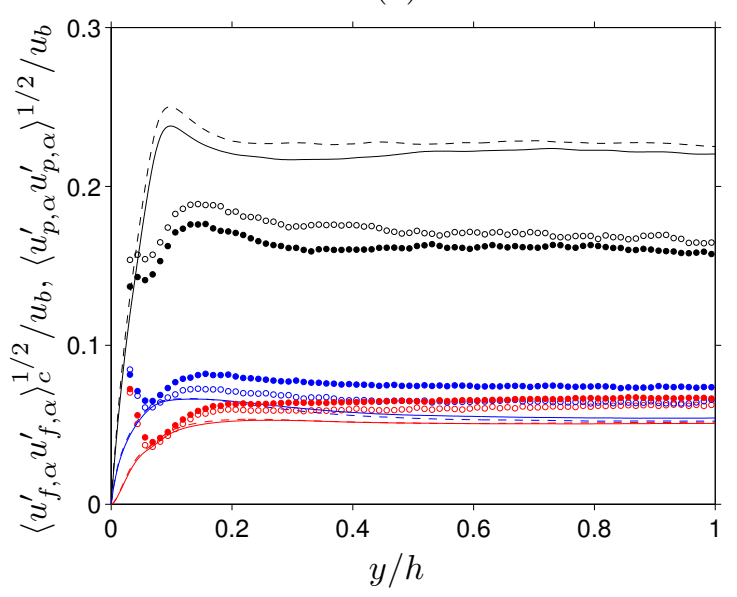

(b)

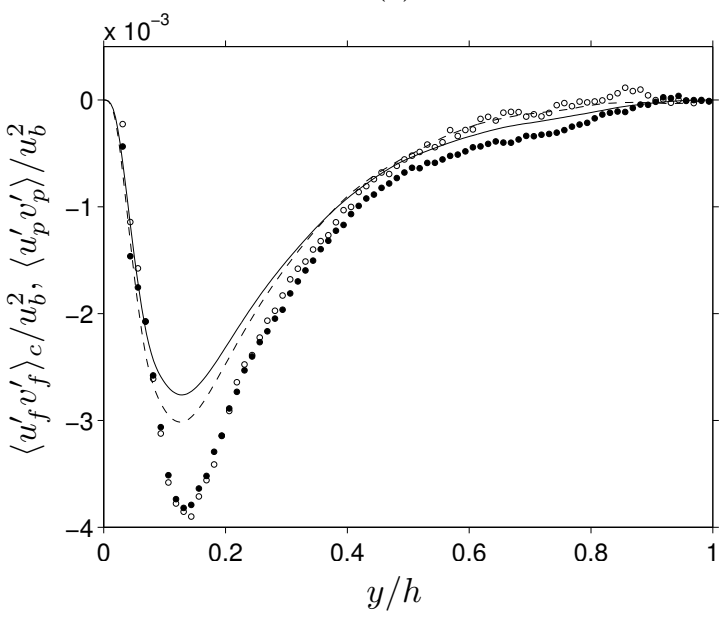

Figure 5: (a) R.m.s. of velocity fluctuations of both phases, with lines corresponding to the fluid phase (○, Uhlmann (2008)), as in figure 3(a). The color coding indicates streamwise (black), wall-normal (red), spanwise (blue) components. (b) Reynolds shear stress of fluid velocity fluctuations as well as corresponding velocity correlation of the particle motion. All quantities are normalized in bulk units. Fluid data shown in this figure is 'composite-averaged' (cf. equation 23) for the purpose of strict comparison; pure fluid averaging data for the present simulation is shown in Appendix B.

\subsubsection{Eulerian statistics of both phases}

Mean fluid and particle velocity profiles as well as particle concentration profiles and the mean spanwise component of the angular particle velocity are shown in figures 3 and 4 . It can be seen that these average values are not too strongly affected by an increase of the box size from $L_{x} / h=8$ to 16 . The observed mostly small differences in these quantities can be attributed to the remaining statistical uncertainty (i.e. limited sampling of the largest flow scales). In particular, the wall shear-stress averaged over the observation interval differs slightly, such that the time-andwall-averaged friction Reynolds number $R e_{\tau}$ takes a value of 220.9 (versus 224.4 in the simulation of Uhlmann, 2008). Furthermore, the previously observed weak tendency towards a concave mean velocity profile (Uhlmann, 2008) is not confirmed by the present results (cf. figure $3 a$ ). It might therefore equally be attributed to limited sampling of the large scales in the previous simulation. The difference between the mean velocities of both phases (cf. figure $3 b$ ) is termed the 'apparent slip velocity', which will be denoted by $u_{\text {lag }}=\left\langle u_{p}\right\rangle-\left\langle u_{f}\right\rangle$ in the following. The corresponding Reynolds number $\left(\operatorname{Re}_{\text {lag }}=\left|u_{\text {lag }}\right| D / \nu\right)$ measures $R e_{\text {lag }} \approx 132$ in the bulk of the channel, while the value drops significantly for wall-distances smaller than $y / h \approx 0.1$.

Concerning the mean value of the spanwise component of angular particle velocity shown in figure 4(b), it should be mentioned that the corresponding graph in Uhlmann (2008) erroneously shows the quantity $\left\langle\omega_{p, z}\right\rangle u_{b} / h$ (i.e. the axis label of figure 14 in Uhlmann, 2008, is incorrect). The correct non-dimensional quantity $\left\langle\omega_{p, z}\right\rangle h / u_{b}$ from that reference is shown in the present figure 4(b). Both datasets exhibit a proportionality as given by $\left\langle\omega_{p, z}\right\rangle=-A \mathrm{~d}\left\langle u_{f}\right\rangle / \mathrm{d} y$ with $A \approx 0.15$, except for the near-wall region $y^{+} \leq 25$. As a consequence, the average shear Reynolds number $R e_{s}=\left|\mathrm{d}\left\langle u_{f}\right\rangle / \mathrm{d} y\right| D^{2} / \nu$ and the average particle rotation Reynolds number $\operatorname{Re}_{\Omega}=\left\langle\omega_{p, z}\right\rangle D^{2} / \nu$ are approximately proportional to each other with the proportionality factor $A$. Incidentally, the particle rotation Reynolds number takes values in the interval $0 \leq R e_{\Omega} \lesssim 1.7$ (not shown), with the maximum occurring at $y / h \approx 0.1$.

Velocity covariances for both phases are shown in figure 5 . It can be observed that the recorded covariance profiles are of very similar values in both simulations, exhibiting only relatively weak differences. In absolute terms, the standard deviation values of the streamwise velocity fluctua- 

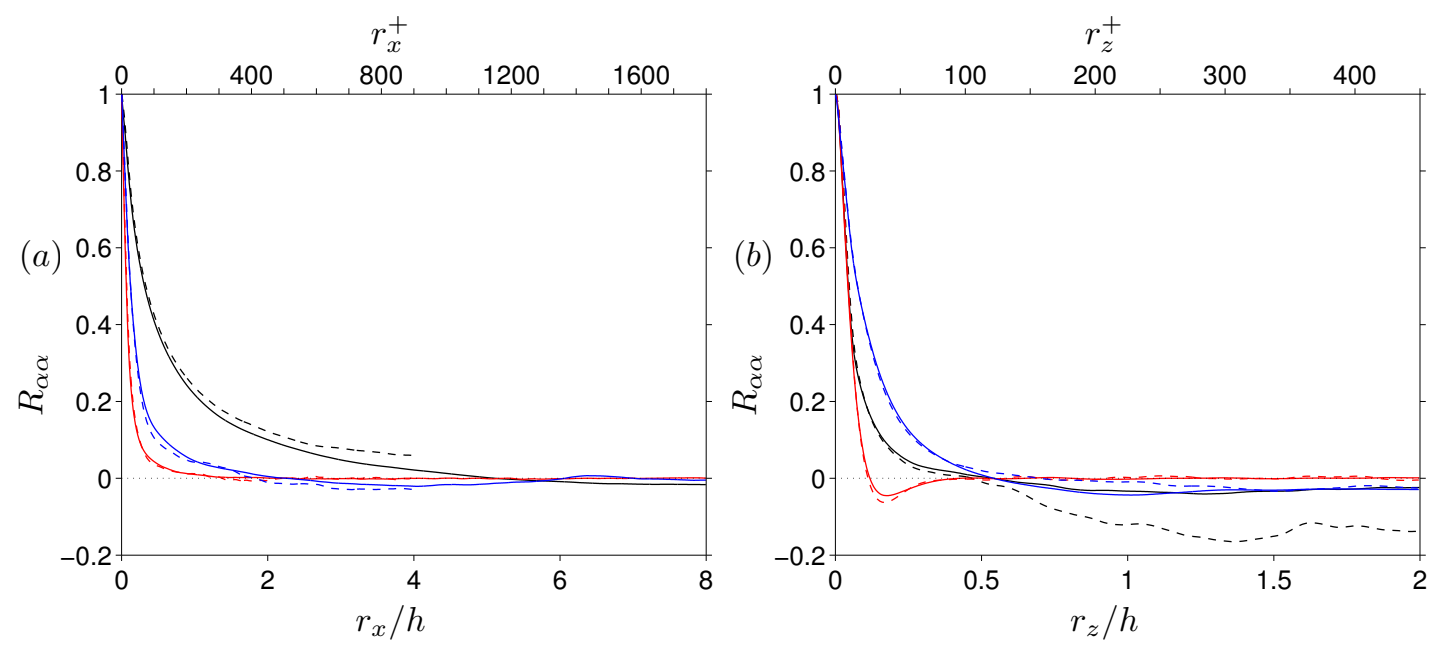

Figure 6: Two-point autocorrelations of fluid velocity fluctuations for $(a)$ streamwise separations and $(b)$ spanwise separations. The correlation functions are evaluated from 12 instantaneous flow fields in the case of Uhlmann (2008) (dashed lines) and from 85 flow fields in the present simulation (solid lines). In both cases only data points in the actual fluid domain are taken into account. streamwise component $(\alpha=1)$, wall-normal $(\alpha=2)$, - spanwise $(\alpha=3)$. The data corresponds to a wall-parallel plane at $y / h=0.0938\left(y^{+} \approx 22\right)$.

tions of both phases $\left(\left\langle u_{f}^{\prime} u_{f}^{\prime}\right\rangle^{1 / 2}\right.$ and $\left.\left\langle u_{p}^{\prime} u_{p}^{\prime}\right\rangle^{1 / 2}\right)$ are reduced on average by $0.007 u_{b}$ and $0.01 u_{b}$, respectively. This reduction is consistent with the higher degree of decorrelation of fluid velocity data in the extended domain, as discussed below. The curves of the wall-normal and spanwise fluid velocity fluctuation intensities in figure $5(a)$ both nearly collapse with their counterparts from the simulation of Uhlmann (2008). On the other hand, the r.m.s. particle velocity fluctuations in the wall-normal and spanwise directions are both slightly larger in the present simulation than in the previous one (on average by $0.005 u_{b}$ and $0.01 u_{b}$, respectively). The profiles of the fluid Reynolds stress (cf. figure $5 b$ ) feature a negative peak of slightly smaller amplitude in the present dataset, while the values of the covariance between streamwise and wall-normal particle velocity fluctuations $\left\langle u_{p}^{\prime} v_{p}^{\prime}\right\rangle$ are approximately the same in both datasets in the vicinity of the negative peak at $y / h \approx 0.15$. Further into the core of the channel (i.e. for $y / h \gtrsim 0.4$ ) the present dataset exhibits slightly higher negative covariance values, both for $\left\langle u_{f}^{\prime} v_{f}^{\prime}\right\rangle$ and $\left\langle u_{p}^{\prime} v_{p}^{\prime}\right\rangle$. The observed differences in the covariance values between the two data-sets is at least in part due to statistical uncertainty, as the previous dataset is found to exhibit somewhat noisier profiles (cf. particularly the curve for $\left\langle u_{p}^{\prime} v_{p}^{\prime}\right\rangle$ in figure $5 b$ ). However, only when additional simulations with a much longer sampling interval are available will it be possible to settle the question about a systematic trend in the fluctuation amplitudes.

Two-point correlations of the fluid velocity field evaluated at a wall-distance of $y / h=0.1$ $\left(y^{+}=22\right)$ are shown in figure 6 . Correlation values for the wall-normal and spanwise velocity components are found to be only very little affected by the prolongation of the domain as the smaller domain size already yielded a reasonable decorrelation at separations of the order of half the fundamental period. On the other hand, fluctuations of the streamwise fluid velocity component decorrelate somewhat more rapidly with streamwise separations in the longer box than they do in the shorter one. Furthermore, the correlation function $R_{11}$ in the present simulation reaches values close to zero at the largest separations $r_{x} / h \approx 8$. Therefore, it makes sense to compute streamwise integral length scales $\left(L_{\alpha \alpha}^{(x)}=\int_{0}^{L_{x} / 2} R_{\alpha \alpha} \mathrm{d} r_{x}\right)$ which take the following values in the present case: $L_{11}^{(x)} / h=0.68, L_{22}^{(x)} / h=0.11, L_{33}^{(x)} / h=0.19$. Concerning spanwise separations, the results from the original and the streamwise-extended domain are similar, with the exception of $R_{11}$ which is visibly less noisy in the present data-set.

Pre-multiplied one-dimensional spectra of the fluid velocity field are shown in figure 7, pro- 
(a)

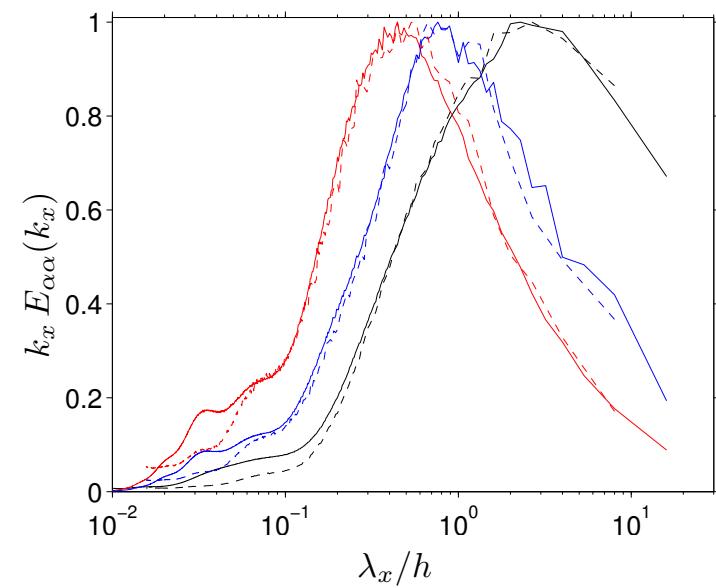

(b)

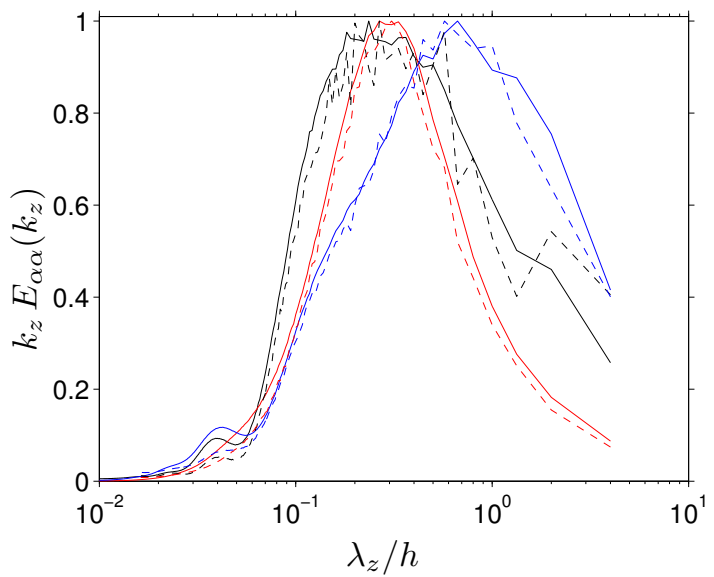

Figure 7: Premultiplied spectra of the three fluid velocity components as function of the wavelength, corresponding to the data in figure 6 (wall-parallel plane at $y^{+}=22$ ). Each curve is normalized to a maximum value of unity in order to emphasize the frequency content. (a) shows streamwise spectra, and $(b)$ spanwise spectra. Line-styles and color-coding as in figure 6 . Note that the particle diameter corresponds to $D / h=5 \cdot 10^{-2}$.

viding an alternative view of the correlation data of figure 6 . In order to compute the spectra, velocity values at nodes inside the particles were set to zero, which leads to the well-known 'stepnoise' at wavelengths around and below the particle scale (Parthasarathy and Faeth, 1990), i.e. at $\lambda / h \approx D / h=0.05$. The large-wavelength end of the spectrum, however, is not affected by the discontinuities at the phase-interfaces. The spectra are normalized by their respective maximum value, in order to enable a comparison of the frequency contents. Concerning the streamwise component of the fluid velocity field, it can be seen in figure 7 that the peak of the energy spectrum is captured both in the longer and shorter boxes. However, the decay with wavelength is more complete in the larger domain. Nevertheless, the data suggest that still a considerably larger streamwise period would be needed in order to allow for a complete energy decay at the largescale end of the spectrum. Unfortunately, a quantitative estimate of the required length cannot be deduced from the present data.

Figures 8 and 9 show streamwise velocity fluctuations from instantaneous fields of simulations in the original domain of Uhlmann (2008) and the extended computational domain of the present simulation. Visual inspection of these and other snapshots corroborates the above finding that the large-scale streak-like structures (first revealed in Uhlmann, 2008) still exist in the present case with a prolonged domain. However, they do not appear to span the entire box length of $L_{x} / h=16$.

\subsubsection{Lagrangian particle velocity correlations}

Uhlmann (2008) found that Lagrangian velocity correlations were strongly affected by the flow structures with the largest streamwise extension. For the present case, figure 10 shows the autocorrelation of particle velocity components along the trajectories. Please note that the correlation data is averaged over all particles and integrated over the full observation interval (cf. definition in equation 7 of Uhlmann, 2008), which means that no distinction is made concerning the particles' instantaneous wall-distance. It had been observed by Uhlmann (2008) that the finite (periodic) domain in conjunction with the presence of very long-lived flow structures leads to a statistical bias manifesting itself in the form of successive peaks in the Lagrangian particle velocity autocorrelation curves at intervals corresponding to an average return time (i.e. equivalent to the domain length $L_{x}$ divided by the apparent velocity lag $u_{r e l} \approx u_{b}$ ). In the present case we still observe repeated peaks in the correlation functions shown in figure 10 , but now at intervals of approximately $16 T_{b}$ - again 
(b)

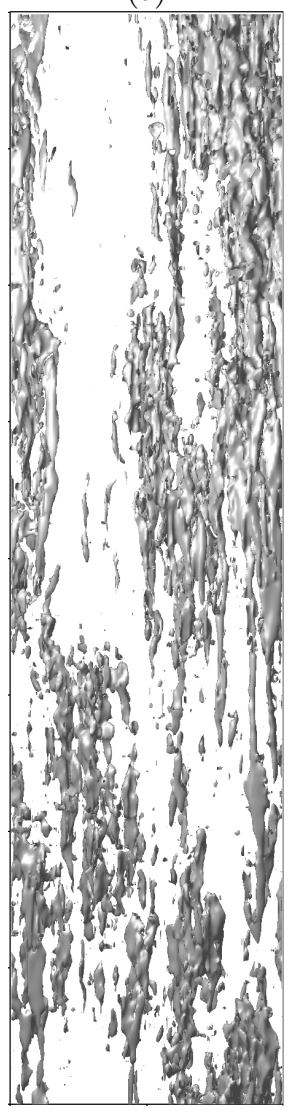

$z / h$

Figure 8: Instantaneous three-dimensional isosurfaces of streamwise velocity fluctuations $u^{\prime}=3.6 u_{\tau}$ (equivalent to $\left.0.3 u_{b}\right)$. The graph $(a)$ corresponds to the simulation in a shorter box Uhlmann (2008), (b) is from the present simulation in a streamwise-elongated box. The view is directed into the wall. Please note that only every eighth grid point in each direction was used.

(b)

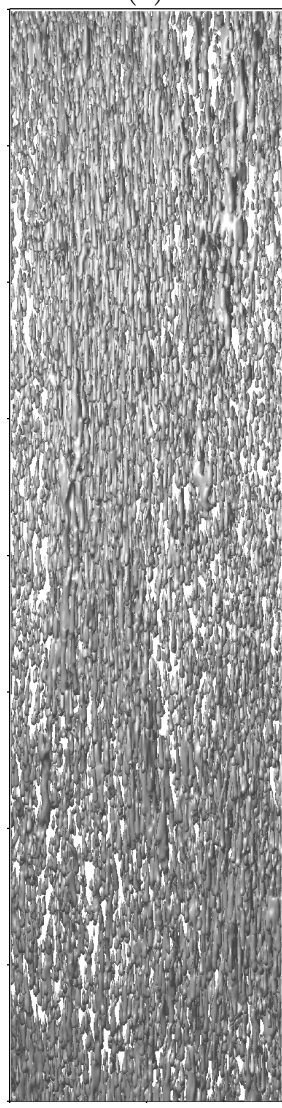

$$
z / h
$$

Figure 9: As figure 8, but showing negativevalued surfaces at the same magnitude, i.e. $u^{\prime}=-3.6 u_{\tau}$. 

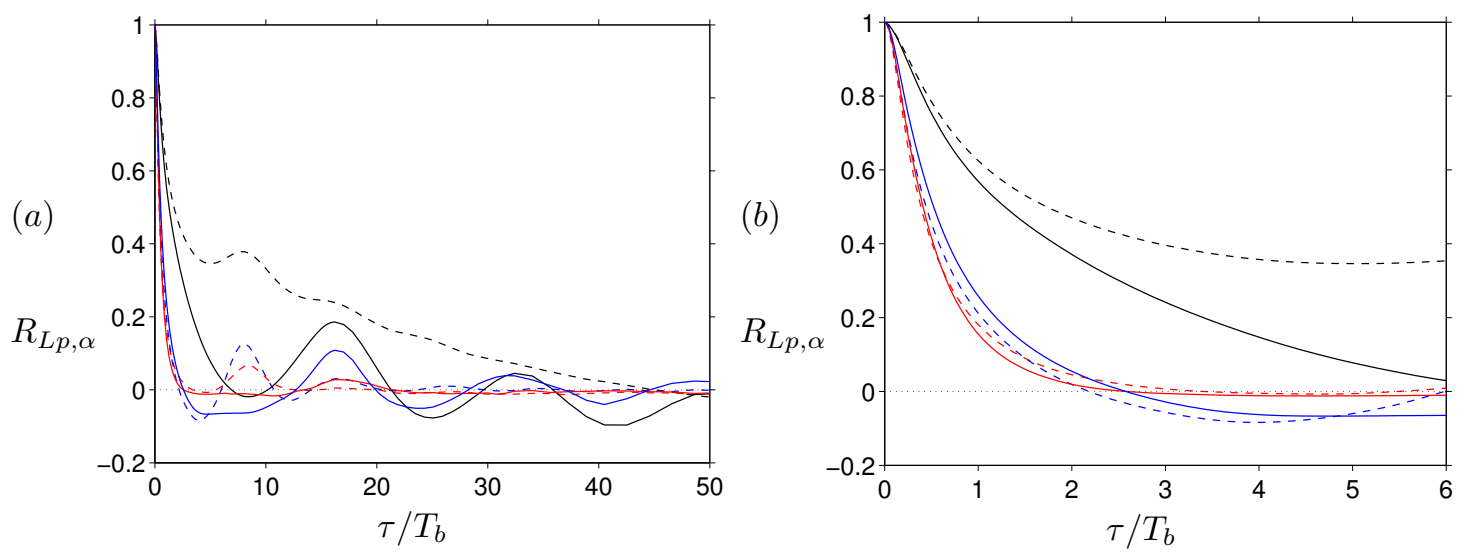

Figure 10: Lagrangian particle velocity autocorrelation as a function of the separation time $\tau$ : streamwise velocity component $(\alpha=1)$; wall-normal $(\alpha=2) ;-$ spanwise $(\alpha=3)$. The results previously obtained in a shorter domain (Uhlmann, 2008) are indicated by dashed lines. The graph in $(b)$ shows a close-up of the same data as $(a)$ for small separation times.

consistent with the expected return time $\left(L_{x} / u_{b}\right)$. Furthermore, a faster initial decorrelation of the streamwise particle velocity component is observed when using the enlarged box. This finding is consistent with the fact that the spatial decorrelation of the corresponding fluid velocity field is faster in the longer box (cf. figure 7), although it is still not complete in the present spatial domain.

Despite the statistical bias due to the finite streamwise period, the short-time behavior of the auto-correlation in the present case is expected to be well represented. As shown in figure 10(b), the initial decay of the correlation function is fastest for the wall-normal component, slightly slower for the spanwise component and significantly slower for the streamwise component of the particle velocity. The corresponding time scale (Taylor micro-scale, i.e. the intercept with the horizontal axis of the osculating parabola at zero separation) measures $0.72 T_{b}, 0.35 T_{b}$ and $0.40 T_{b}$ for the streamwise, wall-normal and spanwise velocity components, respectively. This result is in qualitative agreement with the auto-correlation of fluid particles in single-phase turbulent channel flow (Choi, Yeo and Lee, 2004). The explanation for the directional differences put forth by these authors is based upon the anisotropy of the near-wall coherent structures (velocity streaks and quasi-streamwise vortices). Despite the presence of particle wakes and additional large scales, the flow structure in the present case is similar to single-phase channel flow. Therefore, we expect a similar cause to be responsible for the anisotropic autocorrelation of the particle velocity in the present case.

\subsection{Voronoi analysis of spatial particle distribution}

A number of techniques have been established for the purpose of characterizing the spatial structure of the dispersed phase. In the precursor study (Uhlmann, 2008) conventional box-counting (Fessler, Kulick and Eaton, 1994), nearest-neighbor statistics (Kajishima, 2004), as well as genuine clustering detection algorithms (Wylie and Koch, 2000; Melheim, 2005) were employed. Based upon these measures, it was concluded by Uhlmann (2008) that no significant instantaneous accumulation of particles takes place.

A new technique based upon Voronoi tessellation has recently been proposed in the context of particulate flows (Monchaux et al., 2010; Monchaux, Bourgoin and Cartellier, 2012). Figure 11 shows an example in two dimensions, where starting with given particle center positions (the 'sites') the space is covered with cells which have the property that each point inside the cell is closer to the cell's site than to any other cell's site. As a consequence, the inverse of a Voronoi 

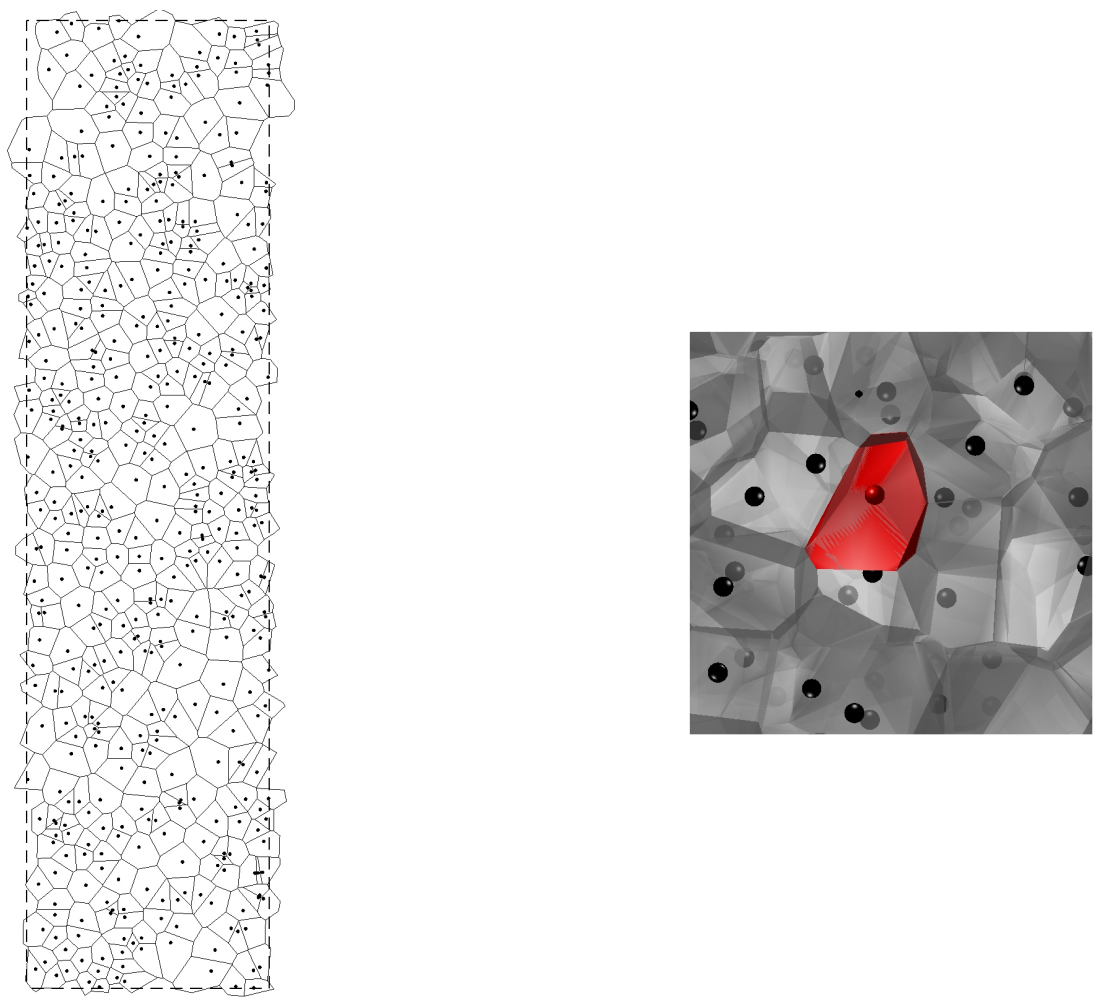

Figure 11: (a) Example of a Voronoi tesselation of a bi-periodic (wall-parallel) plane, performed with respect to the locations indicated as black dots. Note that the cells are continued periodically in order to guarantee a space-filling and non-redundant tesselation. The periodic boundaries are indicated by dashed lines. (b) Close-up of a Voronoi tesselation in three-dimensions, as performed in the present case.

cell's volume is an indicator of the local particle concentration. A statistical analysis of Voronoi tesselations can be performed by computing the p.d.f. of cell volumes, followed by a comparison with reference data for random particle positions (Monchaux et al., 2010, 2012). Compared to previous approaches for characterizing the spatial distribution of the dispersed phase, Voronoi analysis offers two key advantages: (i) richness of geometrical data, offering the possibility to compute various derived diagnostic quantities; (ii) computational efficiency due to the availability of a fast algorithm for the tessellation. In the following, we present the results from an application of this technique to our present data-set.

A random distribution of points in unbounded space yields a Gamma distribution for the Voronoi volumes (Ferenc and Neda, 2007). However, since our particles are of finite size, a random positioning of particle centers without additional constraints can lead to non-physical overlap of particle boundaries. Furthermore, the presence of domain boundaries (walls) can further modify the p.d.f. of Voronoi cell volumes. Therefore, we have numerically determined the Voronoi tessellation of particle positions which have been generated randomly, with the additional constraint that no (particle/particle, particle/wall) overlap is obtained. The corresponding p.d.f.'s (which are indeed close to a Gamma distribution) are added to the graphs for the purpose of comparison with the actual DNS data.

An additional issue arises in the present context due to the spatial inhomogeneity of the average particle concentration. In order to properly account for this variation (i.e. non-constant average volume of Voronoi cells) in the wall-normal direction, we have divided the channel height into 20 intervals of equal width. Statistics of Voronoi cells were then performed individually for each 'bin', i.e. computed over all Voronoi cells whose site is located inside the corresponding wall-normal 
(a)

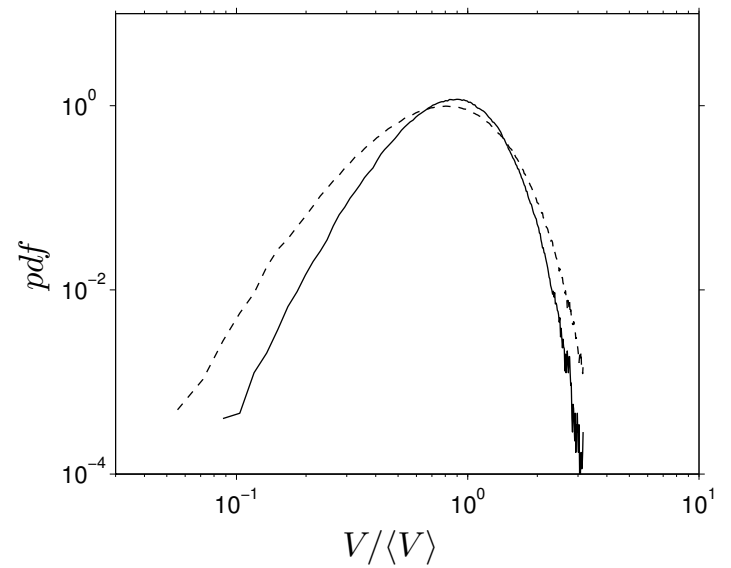

(b)

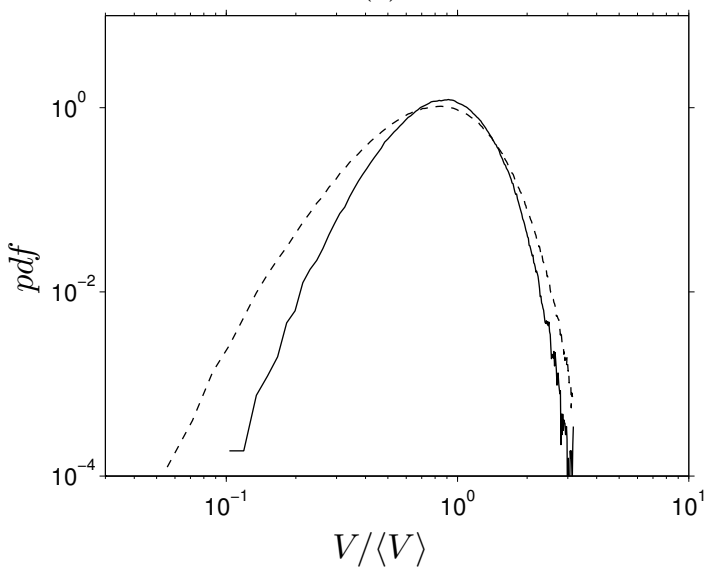

Figure 12: Pdf of Voronoi cell volumes. After three-dimensional Voronoi tesselation, pdfs of the cell volumes are computed in 20 uniform bins across the channel width. The graph in $(a)$ shows the pdf corresponding to the first bin near the wall, $(b)$ is for the bin adjacent to the channel centerline. The present DNS data is shown by a solid black curve; the dashed curve corresponds to randomly placed (non-overlapping) particles. Data is averaged over 2200 instantaneous snapshots.

interval.

A graph of the p.d.f. of Voronoi cell volumes based upon an ensemble of particle positions at 2200 different times in our present simulation is depicted in figure 12, for the interval adjacent to the wall (figure 12a) and for the centerline (figure 12b). In studies of particulate flows which exhibit significant preferential concentration, an appreciable deviation of the Voronoi cell area p.d.f. from the random case is observed (Monchaux et al., 2010), characterized by larger-thanrandom probabilities of finding very small and very large Voronoi cells while roughly maintaining the overall shape of the p.d.f.. Contrarily, our data in figure 12 shows the opposite behavior: very small Voronoi cells and very large Voronoi cells are less probable than in the case of randomly placed particles. In other words, large deviations from the average cell volume are found to be less probable than in a case of randomly placed particles. Therefore, the state of the dispersed phase can be characterized as more ordered than random, slightly (but significantly) tending towards a homogeneous spatial distribution. It should be noted that the results of Uhlmann (2008) for the average distance to the nearest particle (cf. figure 23 therein) did indicate a slight deviation from a fully random particle ensemble, tending towards the value for a homogeneous particle array. Box-counting and direct cluster identification, however, did not pick up noticeable differences with respect to randomness.

The deviation from a random state as presently observed from Voronoi cell volume statistics is consistent with particles having a weak tendency to form a regular pattern. While the extreme case of particles forming a uniform cubical lattice would yield a Dirac distribution (with a pulse at the average cell volume), the present data shown in figure 12 only weakly deviates from the purely random reference curve. On the other hand, preferential particle concentration outside of vortical structures can indeed be ruled out in the present case, confirming the previous findings of the precursor study (Uhlmann, 2008).

Additional quantities of interest can be deduced from the Voronoi tessellation. In our case, where there exist preferred spatial directions due to gravity and the general non-isotropy of channel flow, particle accumulation (if any) is not expected to take place in an isotropic manner. In particular, possible elongated particle chains with a preferential orientation can be expected to lead to non-isotropic statistics of Voronoi cells. Therefore, we have computed the aspect ratio of Voronoi cells dividing the largest streamwise extension $l_{x}$ by the largest wall-normal $l_{y}$ and 
(a)

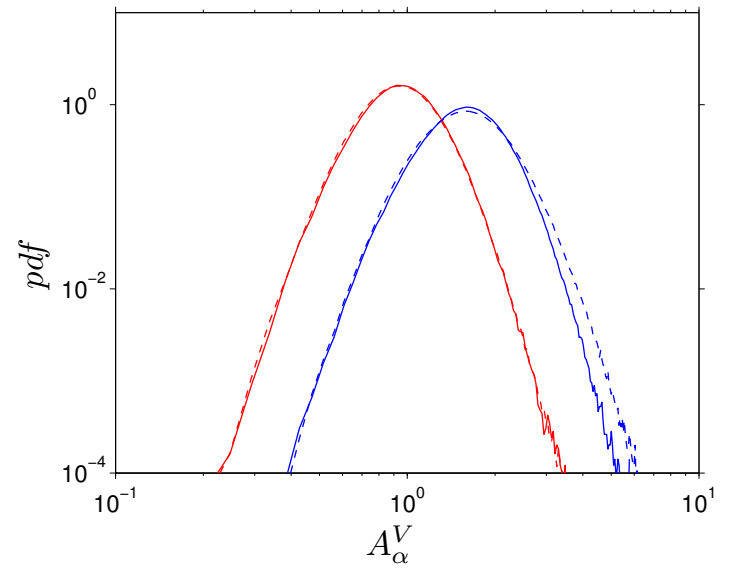

(b)

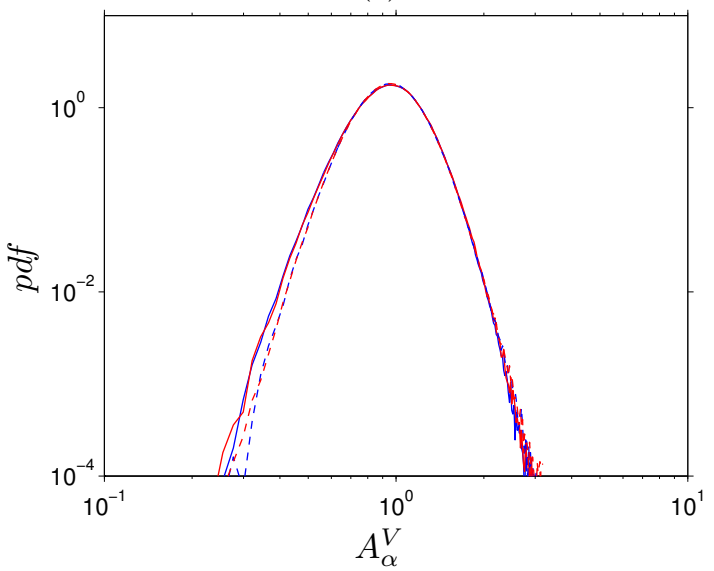

Figure 13: Pdf of Voronoi cell aspect ratios, defined as the maximum extension of a given cell in the respective coordinate directions: streamwise/wall-normal $\left(A_{y}^{V}\right)$ are shown in blue, streamwise/spanwise $\left(A_{z}^{V}\right)$ are shown in red. As in figure 12, graph $(a)$ shows the pdf corresponding to the first bin (out of 20 ) near the wall, $(b)$ is for the bin adjacent to the channel centerline; solid lines correspond to DNS data, dashed lines to random particle positions.

spanwise extensions $l_{z}$ of each Voronoi cell, viz.

$$
A_{\alpha}^{V}=\frac{l_{x}}{l_{\alpha}} \quad \forall \alpha=2,3 .
$$

The corresponding p.d.f.'s are shown in figure 13 (again for two different wall-normal intervals). Adjacent to the solid surface, the streamwise/spanwise and the streamwise/wall-normal aspect ratios are clearly distinct: the latter having a mean of 1.74 due to the constraint by the wallboundary. In the center of the channel the constraint by the wall is no longer felt and the curves for the streamwise/spanwise and the streamwise/wall-normal aspect ratios coincide. Except for the first interval adjacent to the walls, deviations of the DNS data from the curves for random particle arrangements are only observed as an increased probability of very small values $A_{y}^{V}$ and $A_{z}^{V}$, i.e. Voronoi cells which are squeezed in the streamwise direction are found more frequently than in a random arrangement. This small but systematic difference (cf. figure 13b) implies that particles have indeed a weak tendency to align along the streamwise direction. It can be speculated that such an alignment is induced by the sheltering effect of particle wakes, which is known to cause trailing particles to approach leading particles (Wu and Manasseh, 1998; Fortes, Joseph and Lundgren, 1987).

\subsection{Particle acceleration statistics}

In this section, we study the Lagrangian acceleration statistics of the solid particles in the present flow. In recent years much attention has been given to the Lagrangian acceleration statistics of fluid and solid particles, see for example the review by Toschi and Bodenschatz (2009). Apart from being relevant to a number of applications, the growing interest has been mainly due to the appearance of new experimental methods and the abundance of direct numerical simulations employing the pointparticle approach. Most previous studies have been performed for homogeneous flows. Exceptions are, for example, the study of Lagrangian acceleration statistics of sub-Kolmogorov size inertial particles in a turbulent boundary layer, by means of experiments (Gerashchenko et al., 2008) and DNS (Lavezzo, Soldati, Gerashchenko, Warhaft and Collins, 2010). Finite size effects on Lagrangian particle acceleration statistics have mainly been studied experimentally, using both neutrally buoyant and heavy particles (Qureshi et al., 2007, 2008; Xu and Bodenschatz, 2008; Brown et al., 2009). Two contributions based upon DNS studies should be mentioned. Calzavarini 


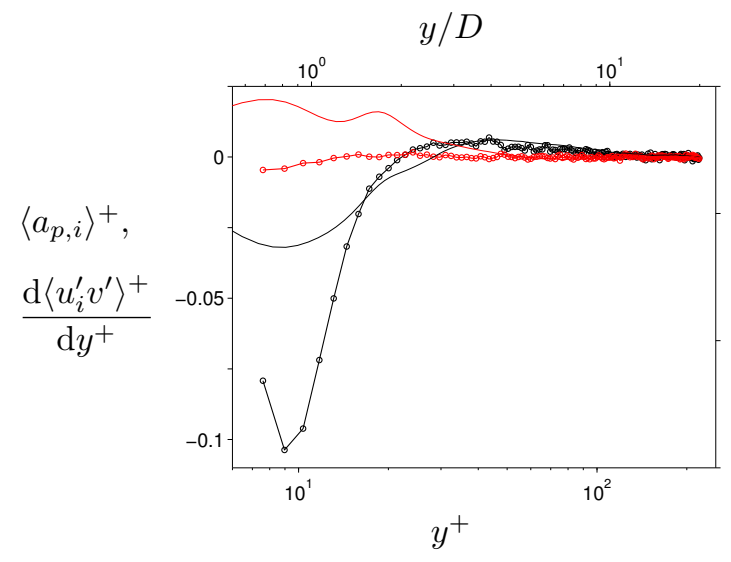

Figure 14: Mean particle acceleration, as a function of the wall distance. -o-, streamwise component $\left\langle a_{p, 1}\right\rangle$; - - , wall-normal component $\left\langle a_{p, 2}\right\rangle$. The lines without symbols show the wall-normal gradients of Reynolds stresses: -, $\mathrm{d}\left\langle u^{\prime} v^{\prime}\right\rangle / \mathrm{d} y ;-, \mathrm{d}\left\langle v^{\prime} v^{\prime}\right\rangle / \mathrm{d} y$. Wall units have been used as the normalization scale $\left(a_{\text {ref }}=u_{\tau}^{3} / \nu\right)$.

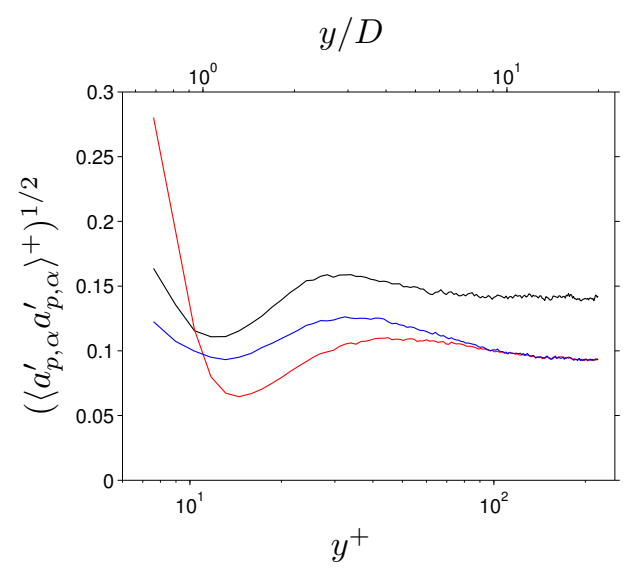

Figure 15: Standard deviation of particle acceleration as a function of the wall distance. — , streamwise component; —_ , wall-normal component; ——, spanwise component.

et al. (2009) have extended the point-particle approach by including Faxén corrections, while Homann and Bec (20010) have simulated the motion of a single, resolved, finite-size particle, both in forced homogeneous-isotropic turbulence. In both cases, however, there was no apparent slip velocity due to the exclusion of gravity in the former case and due to matched density in the latter.

Before turning to the present results, it should be pointed out that we have filtered from the particle force data all records corresponding to particle collisions (i.e. those where there is a force contribution $\mathbf{F}_{C}$ in equation 1a stemming from the artificial repulsion force).

Since the present flow is inhomogeneous in the wall-normal direction, we start by considering the mean Lagrangian acceleration of the particles, a quantity which is zero in statistically stationary homogeneous flows. Recall that in turbulent channel flow the mean streamwise (wall-normal) Lagrangian acceleration of fluid particles turns out to be equal to the wall-normal gradient of $\left\langle u_{f}^{\prime} v_{f}^{\prime}\right\rangle\left(\left\langle v_{f}^{\prime} v_{f}^{\prime}\right\rangle\right)$ (Yeo, Kim and Lee, 2010). Fig. 14 displays the mean streamwise $\left\langle a_{p 1}\right\rangle$ and wallnormal $\left\langle a_{p 2}\right\rangle$ acceleration of the solid particles. Also for comparison the mean streamwise and wall-normal Lagrangian fluid particle accelerations $\left\langle a_{f 1}\right\rangle=\partial_{y}\left\langle u_{f}^{\prime} v_{f}^{\prime}\right\rangle$ and $\left\langle a_{f 2}\right\rangle=\partial_{y}\left\langle v_{f}^{\prime} v_{f}^{\prime}\right\rangle$ are shown. The streamwise and wall-normal acceleration components are non-zero near the wall, while the mean spanwise acceleration is zero everywhere (not shown). In the central region, $\left\langle a_{p 1}\right\rangle$ is roughly zero, as can be expected from an equilibrium between buoyancy and drag forces. The presence of the wall alters this equilibrium and a negative peak in $\left\langle a_{p 1}\right\rangle$ appears at $y^{+} \approx 10$, which is significantly larger in magnitude than the corresponding peak observed for $\left\langle a_{f 1}\right\rangle$. Furthermore, the mean particle acceleration $\left\langle a_{p 1}\right\rangle$ presents a milder positive peak at $y^{+} \approx 30$. Concerning the wall-normal acceleration, $\left\langle a_{p 2}\right\rangle$ exhibits slightly positive values in the interval $15 \lesssim y^{+} \lesssim 40$ (equivalent to a mean lift acting towards the channel center) which are smaller in magnitude than those presented by the fluid counterpart $\left\langle a_{f 2}\right\rangle$. Closer to the wall $\left(y^{+} \lesssim 15\right)$ the mean wallnormal particle acceleration takes slightly negative values. The peak in $\left\langle a_{p 1}\right\rangle$ might be explained by the following mechanism. Similar to turbulent fluid motion, inertial particle motion near the wall exhibits a preference for Q2 and Q4 events (in the terminology of quadrant analysis of the streamwise and wall-normal velocity fluctuations, where Q2 refers to ejections of low-speed fluid away from the wall and Q4 to an inrush - or sweep - of high-speed fluid), as shown by Uhlmann (2008). As a consequence, significant average values of the cross-correlation (i.e. 'Reynolds stress') 
(a)

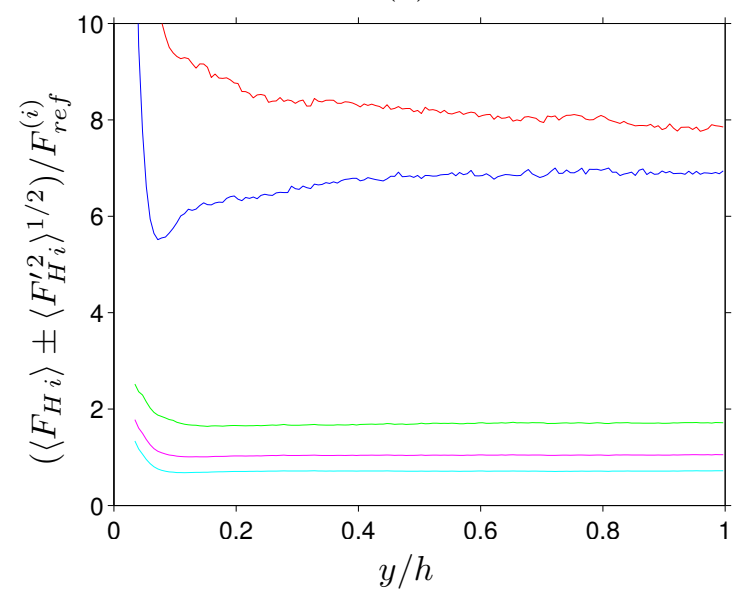

(b)

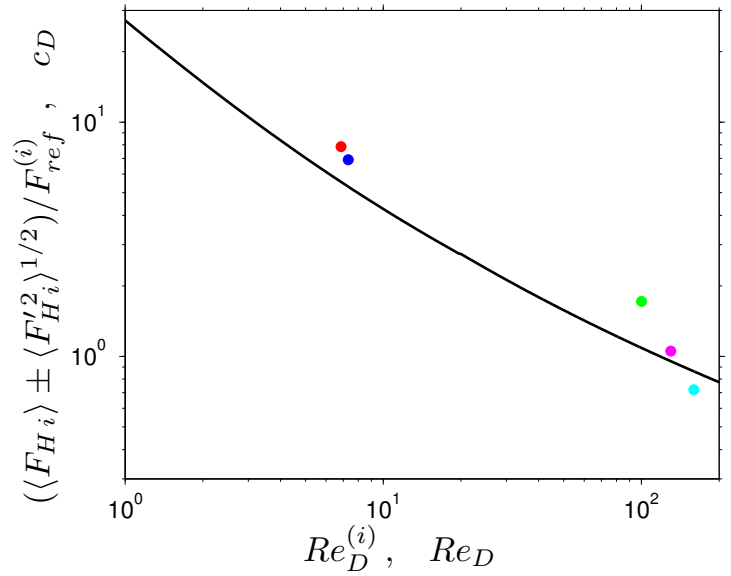

Figure 16: Mean and rms hydrodynamic particle force components normalized by a reference force $F_{r e f}^{(i)}=\frac{1}{2} \rho_{f} A_{p} u_{r e l}^{(i) 2}$ (where $A_{p}=\pi D^{2} / 4$ ). (a) shows wall-normal profiles, while in $(b)$ the data is presented as a function of the local Reynolds number based on an approximation for the relative velocity. The red (blue) line is for the rms particle force in the wall-normal (spanwise) direction, the relative velocity in the reference force being taken as the rms fluid velocity, i.e. $u_{\text {rel }}^{(2)}=\left\langle v_{f}^{\prime} v_{f}^{\prime}\right\rangle^{1 / 2}\left(u_{r e l}^{(3)}=\left\langle w_{f}^{\prime} w_{f}^{\prime}\right\rangle^{1 / 2}\right)$. The magenta-colored line indicates the average particle force in the streamwise direction, the relative velocity being taken as the mean apparent slip velocity $u_{r e l}^{(1)}=\left\langle u_{p}\right\rangle-\left\langle u_{f}\right\rangle$. The cyan (green) colored lines indicate the mean plus (minus) the rms particle force in the streamwise direction, normalized by a reference force based upon the apparent slip velocity plus (minus) the rms streamwise fluid velocity. Concerning the graph in (b): the horizontal axis represents the Reynolds number based upon the particle diameter and the respective relative velocity $u_{r e l}^{(i)}$. The black solid line indicates the standard drag law for spheres in uniform flow (Clift et al., 1978, table 5.2). The symbols correspond to the data in $(a)$ on the channel centerline, using the same color coding.

$\left\langle u_{p}^{\prime} v_{p}^{\prime}\right\rangle$ arise. Since the particles have larger inertia than a corresponding blob of fluid, it can be expected that it takes longer for the particles to adjust to the surrounding fluid conditions, and consequently the correlation values can be expected to exceed those of the fluid counterpart, as is indeed the case (cf. figure $32 b$ ). Now, since particles arriving in the near-wall region from larger wall-distances carry on average an excess axial velocity value (Q4 events), these particles will tend to experience a smaller amount of positive streamwise force (drag). As a consequence, the (negative) submerged weight will exceed the positive drag and a negative particle acceleration in the vertical direction will result. The opposite is expected for particles being ejected away from the wall (Q2 events). The location where the average acceleration changes sign from negative values to positive ones $\left(y / h \approx 0.1, y^{+} \approx 20\right)$ coincides approximately with the location where the gradient of the Reynolds shear stress changes sign; it is also an upper bound for the near-wall region in which appreciable wall-normal gradients of the wall-normal particle velocity fluctuation energy $\left\langle v_{p}^{\prime} v_{p}^{\prime}\right\rangle$ exist (cf. figure 32). As a consequence, the turbophoretic effect (Caporaloni et al., 1975; Reeks, 1983; Uhlmann, 2008) can be expected to lead to a preponderance of negative particle acceleration for $y / h \lesssim 0.1$, as observed in figure 14 .

Fig. 15 shows the r.m.s. values of the three components of the particle acceleration scaled in wall units. The values obtained are comparable in magnitude to the r.m.s. fluid particle acceleration in single-phase channel flow (Yeo et al., 2010) and to the r.m.s. acceleration of inertial particles in a turbulent boundary layer (Gerashchenko et al., 2008). The three components present a similar trend: a peak near the wall, a dip in the buffer layer and a gradual increase towards a mild maximum and finally a plateau in the central region. The r.m.s. of streamwise acceleration is larger than the other two components by about $50 \%$ except near the wall $\left(y^{+}<10\right)$, where the 
$(a)$

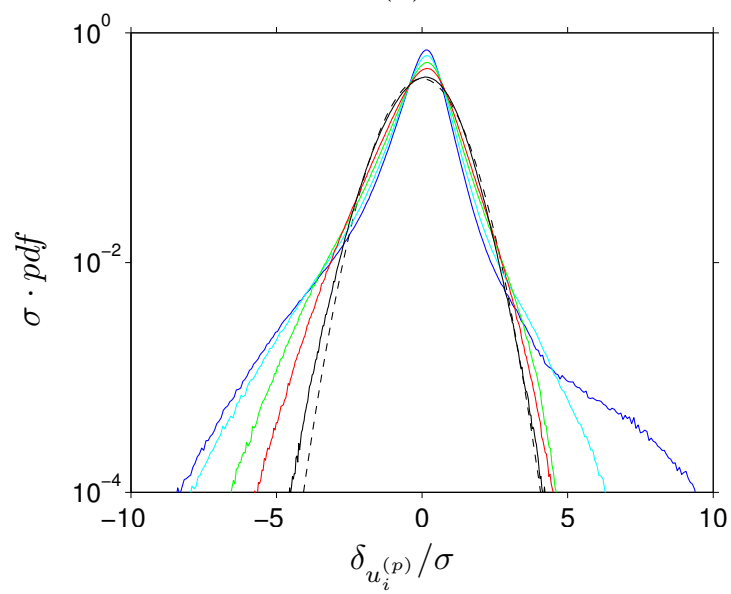

(b)

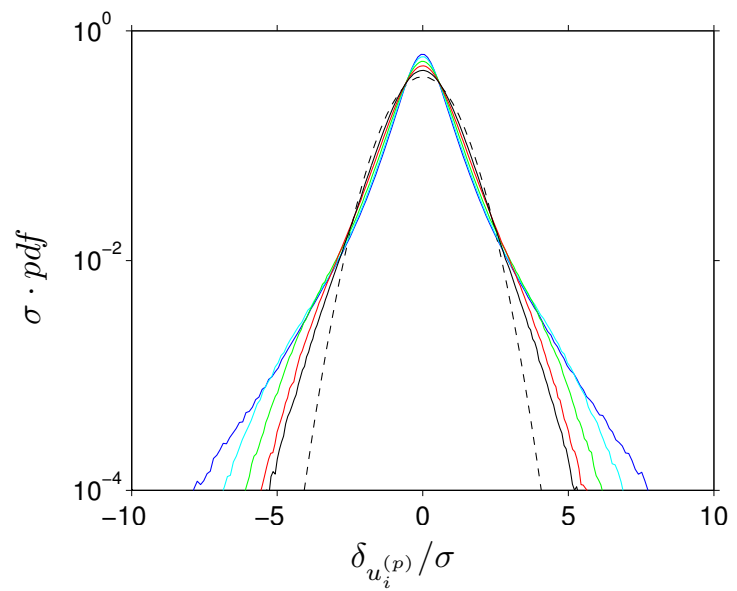

Figure 17: Normalized probability density function of Lagrangian particle velocity increments $\delta_{u_{p, i}}=u_{p, i}^{\prime}(t+\tau)-u_{p, i}^{\prime}(t)$ in $(a)$ the streamwise direction $(b)$ the wall-normal direction. The different curves correspond to various values of the time lag $\longrightarrow, \tau^{+}=1.4 ;-, \tau^{+}=2.9$; ,$\tau^{+}=7.2 ;-, \tau^{+}=14.3 ;-, \tau^{+}=70.8$.

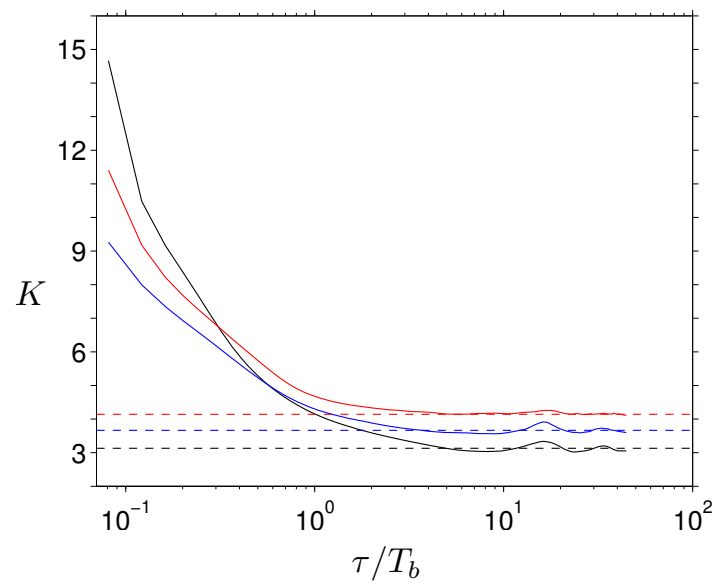

Figure 18: Kurtosis $K\left(\delta_{u_{p, i}}\right)$ of the Lagrangian velocity increments of solid particle velocities $\delta_{u_{p, i}}(\tau)=u_{p, i}^{\prime}(t+\tau)-u_{p, i}^{\prime}(t)$, plotted as a function of the separation time $\tau$. The three coordinate directions are color-coded as in figure 15. For each component the dashed line indicates the asymptotic value $K\left(u_{p, i}^{\prime}\right) / 2+3 / 2$.

r.m.s. of the wall-normal component presents maximum values.

Further insight can be obtained by studying the r.m.s. of the hydrodynamic forces on the particles, since by Newton's law studying the force is equivalent (up to a constant coefficient) to studying the acceleration. We distinguish here the streamwise direction and the transverse directions. For the streamwise direction the mean drag force is approximately balanced by the gravity force, while for the transverse directions the mean lift and spanwise forces are zero, except for the lift very near the wall, as discussed above. We consider, therefore, for the transverse directions the r.m.s. of the hydrodynamic force and for the streamwise direction we consider first, the mean hydrodynamic force, and second the mean hydrodynamic force plus (or minus) the r.m.s. of the hydrodynamic force. For each direction, we use as a reference force $F_{r e f}^{(i)}=\frac{1}{2} \rho_{f} A_{p} u_{r e l}^{(i) 2}$ where $A_{p}=\pi D^{2} / 4$ and $u_{r e l}^{(i)}$ is a reference velocity characteristic for the relative flow in the $i$ th coordinate direction. We estimate the relative velocity scale as follows: for the transverse 

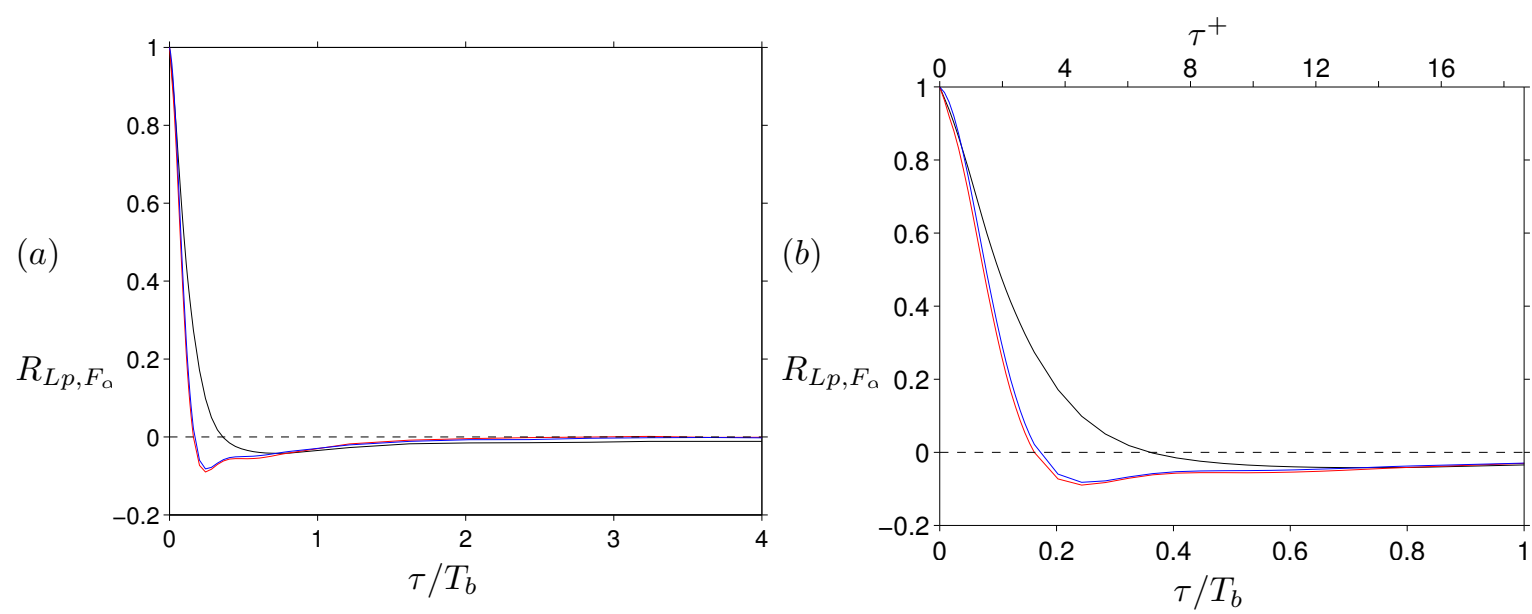

Figure 19: Lagrangian autocorrelation of hydrodynamic force acting upon the particles, plotted as a function of the separation time $\tau$ : —, streamwise $(\alpha=1)$, $\longrightarrow$, wall-normal $(\alpha=2)$, - , spanwise $(\alpha=3)$ components. The graph in $(b)$ shows a close-up of the same data as $(a)$ for small separation times, including scaling of the abscissa in bulk time units and in wall units.

directions the r.m.s. fluid velocity is employed, viz. $u_{r e l}^{(2)}=\left\langle v_{f}^{\prime} v_{f}^{\prime}\right\rangle^{1 / 2}$ and $u_{r e l}^{(3)}=\left\langle w_{f}^{\prime} w_{f}^{\prime}\right\rangle^{1 / 2}$; in the streamwise direction, the relative velocity is taken as the mean apparent slip velocity (plus or minus the r.m.s. fluid velocity), $u_{\text {rel }}^{(1)}=u_{\text {lag }}$ ( or $u_{r e l}^{(1)}=u_{\text {lag }} \pm\left\langle u_{f}^{\prime} u_{f}^{\prime}\right\rangle^{1 / 2}$ ), according to whether the mean or the mean plus/minus the r.m.s. value of the hydrodynamic force are considered. Using this normalization, the mean and r.m.s. hydrodynamic forces are shown in Fig. 16(a). All curves are rather flat for $y / h>0.2$, and they can be interpreted as force coefficients. Defining a particle Reynolds number $R e_{D}^{(i)}$ in each direction based on the respective relative velocity $u_{r e l}^{(i)}$, and plotting the force coefficients from the central region of the channel as a function of $R e_{D}^{(i)}$, as shown in Fig. 16(b), it turns out that the force coefficients are consistent with the standard drag law for spheres in uniform flow (Clift et al., 1978, table 5.2). We have checked that the scaling used for the transverse direction leads in the case of the streamwise direction to a force coefficient which is not consistent with the standard drag law. As might be expected, the interpretation of the normalized hydrodynamic force in terms of a drag coefficient (as shown in Fig. 16b) does provide an explanation for the large differences in r.m.s. aceleration/force observed when simply normalizing all components in wall units (cf. Fig. 15). However, given the rather crude assumptions it is indeed remarkable that the mean streamwise force coefficient in Fig. $16 b$ turns out relatively close to the value given by the standard drag law valid for a fixed sphere in uniform flow.

Next we consider the p.d.f.'s of Lagrangian particle velocity increments $\delta_{u_{p, i}}(\tau)=u_{p, i}^{\prime}(t+$ $\tau)-u_{p, i}^{\prime}(t)$. Fig. 17 displays the p.d.f.'s of streamwise and wall-normal increments for various time lags. Similar graphs have been reported by Mordant et al. (2002) and Qureshi et al. (2007), among others. The figure shows that for increasing time increments the p.d.f.'s become more like Gaussian distributions. For short time increments, of the order of the viscous time scale, pronounced tails are present, which are the signature of intermittent Lagrangian dynamics. It is interesting to note the skewness that appears in the p.d.f. corresponding to the streamwise velocity increments, a feature that has not been observed in previous investigations in other flow geometries (Mordant et al., 2002; Qureshi et al., 2007). This point will be further discussed below. The decrease of the tails of the velocity increment p.d.f.'s with increasing separation time $\tau$ can be gauged by examining the temporal evolution of the kurtosis of $\delta_{u_{p, i}}(\tau)$, as shown in figure 18 . It can be seen that the initial decrease is fastest for the streamwise direction, while the curves for the two horizontal directions are similar to each other, but with a slight shift in the value of the kurtosis. The three components exhibit slight 'bumps' at multiples of $16 T_{b}$ corresponding to the finite-box-size bias (cf. discussion in $\S 3.1 .2$ ), the curves reaching asymptotic values which 


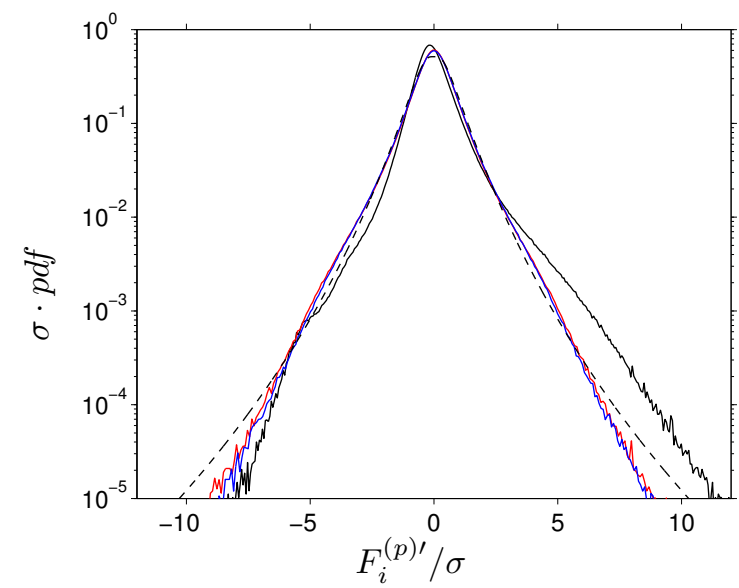

Figure 20: Normalized probability density function of hydrodynamic forces acting on the particles, averaged over the full channel height,___, streamwise force component; —_, wall-normal component; —_, spanwise component; ----, log-normal fit proposed in Qureshi et al. (2008).

(a)

$y / D$

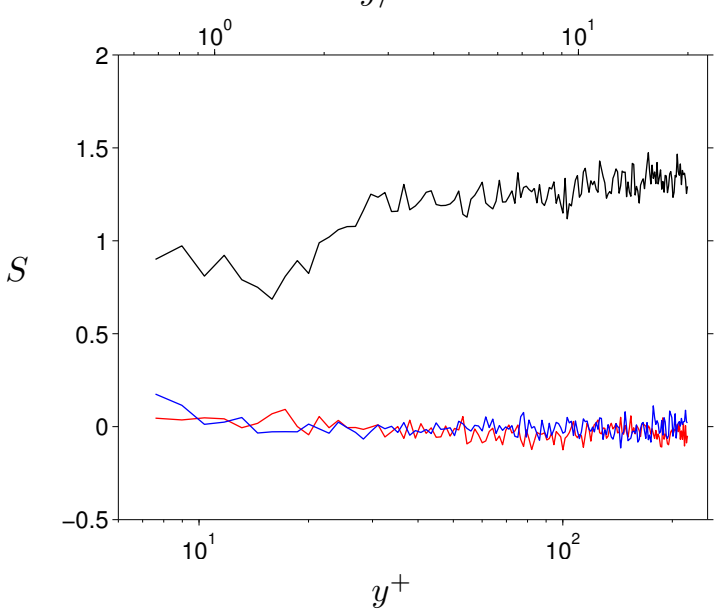

$(b)$

$y / D$

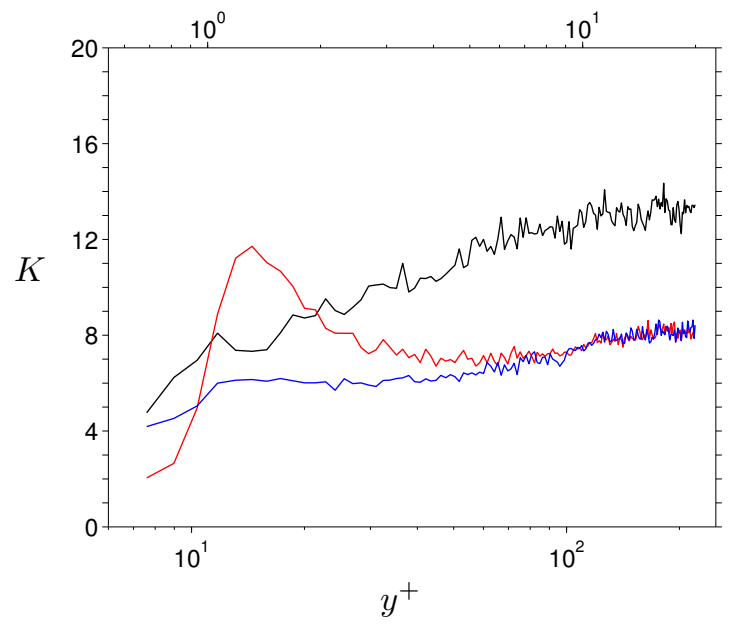

Figure 21: Higher-order moments of particle acceleration: $(a)$ skewness $S,(b)$ kurtosis $K$. Lines as in figure 15.

are close to Gaussian for the streamwise direction $\left(K\left(\delta_{u_{p}}\left(\tau=40 T_{b}\right)=3.0\right)\right.$ and somewhat larger for the two horizontal directions $\left(K\left(\delta_{v_{p}}\left(\tau=40 T_{b}\right)=4.2\right.\right.$ and $K\left(\delta_{w_{p}}\left(\tau=40 T_{b}\right)=3.6\right)$. It can be shown that if the signals at time $t$ and $t+\tau$ are completely decorrelated, the kurtosis of the increment will have a value of $K\left(u_{p, i}^{\prime}\right) / 2+3 / 2$ (note that this limit is indeed approached by our data at long times as can be seen in figure 18). As a consequence, the different long-time limits of $K\left(\delta_{u_{p, i}}\right)$ reflect the differences with respect to intermittency of the three components of the particle velocity (cf. the one-time p.d.f.'s shown in figure 17 of Uhlmann, 2008).

Let us now turn to the analysis of correlation times of the Lagrangian statistics. Fig. 19 shows the Lagrangian autocorrelation of the hydrodynamic forces acting on the particles (which is equivalent to the autocorrelation of particle acceleration). It can be observed that the autocorrelation functions first cross the zero-axis after several viscous time units (the streamwise component after $7 \nu / u_{\tau}^{2}$, the wall-normal and spanwise components after approx. $3 \nu / u_{\tau}^{2}$ ), then taking small negative values which return to zero on a longer time scale of the order of $2 T_{b}$. The initial decay can be characterized through the Taylor micro-scale, which is found to measure 2.95, 0.92 and 0.80 
viscous time units for the streamwise, wall-normal and spanwise force components, respectively. The figure shows that although the initial decorrelation strongly differs among the three force components, their long-time behavior (for separation times $\tau / T_{b} \gtrsim 0.7$ ) is roughly equivalent. A very similar directional dependence of the Lagrangian autocorrelation at short separation times has also been observed for the acceleration of fluid particles in single-phase channel flow (Choi et al., 2004). As in the case of Lagrangian velocity autocorrelations (discussed in $\S 3.1 .2$ ), these authors attribute the effect to the anisotropy of the near-wall coherent structures. In view of the striking similarity between the present data (figure 19b) and the fluid particle data (figure 13, Choi et al., 2004), we believe that the presently observed difference in the initial decorrelation time scale can be attributed to the anisotropic nature of the wall-bounded turbulent flow scales.

In the limit of very short time increments, the p.d.f.'s of Lagrangian particle velocity increments, discussed above, become the p.d.f.'s of Lagrangian particle acceleration. Qureshi et al. (2008) have shown that acceleration statistics of finite size inertial particles are found very robust to size and density variations, the influence of which is mostly carried by the acceleration variance. They found that the shape of the normalized p.d.f. remained unchanged over the whole range of sizes and density ratios explored. The p.d.f.'s shown in Fig. 20 confirm this result in the present case for the transverse directions (wall-normal and spanwise). Both curves follow the lognormal fit proposed by Qureshi et al. (2008) relatively closely over several decades. On the other hand, the streamwise acceleration deviates from this curve, showing significantly higher probabilities for positive (upward) acceleration fluctuations. It is not clear why this p.d.f. is positively skewed. One possible mechanism that provides positive skewness is as follows. Assume that a non-linear drag law holds instantaneously, i.e. $C_{d}=f\left(R e_{i n s t}\right)$, where $R e_{i n s t}$ is based on the instantaneous relative velocity felt by the particle, and that the drag coefficient decays more slowly with Reynolds than $R e_{\text {inst }}^{-1}$. In such a case, positive velocity fluctuations would lead to a smaller decrease in the drag coefficient than the corresponding increase due to negative velocity fluctuations of the same magnitude. As a consequence the drag force (as well as the corresponding acceleration) can become positively skewed even when the velocity fluctuations are symmetric w.r.t. the mean. The data in Fig. 16(a) is consistent with this model: it can be seen that the streamwise force coefficient obtained using the mean drag plus the r.m.s. drag is closer to the force coefficient of the mean drag than the force coefficient obtained using the mean drag minus the r.m.s. drag. We have further tested the hypothesis based upon non-linear drag by using a Gaussian relative velocity p.d.f. as input to the standard drag law. Although this simple model indeed yields positively skewed hydrodynamic forces (figure omitted), the magnitude of the skewness obtained is smaller than the one obtained in the simulation. Therefore, this issue deserves further investigation.

The force/acceleration p.d.f.'s shown in Fig. 20 have been computed without taking into account the inhomogeneous nature of the flow. In order to illustrate how the wall affects the p.d.f.'s we have also computed them in 160 wall-normal slabs of uniform thickness. It suffices to discuss the wall-normal variation of the p.d.f.'s in terms of their higher order moments, of which skewness and kurtosis are shown in Fig. 21. Only the streamwise component presents significant positive skewness which varies from $S \approx 0.8$ close to the wall to $S \approx 1.3$ in the central region. The wallnormal and spanwise force/acceleration components are roughly symmetrically distributed, with the exception of the region near the wall where a small positive skewness is obtained. Concerning the kurtosis, all three components tend to present higher values in the central region of the channel where the streamwise component is larger than the other two components by approximately $60 \%$. This indicates that the tails of the p.d.f. of the streamwise component are broader as can be appreciated in Fig. 20. The kurtosis of the wall-normal and spanwise components are equal in the central region of the channel, indicating that in this zone these two directions are equivalent as mentioned above. When approaching the wall, they deviate from each other. The kurtosis of the spanwise component remains more or less constant at a value of $K \approx 6$ up to the immediate near-wall region where it decreases mildly. The kurtosis of the wall-normal component, on the other hand, presents a remarkable peak in the buffer layer (with $K \approx 12$ ) which is even higher than the kurtosis of the streamwise component in this region. Closer to the wall the kurtosis of the wall-normal component tends towards a Gaussian value. 


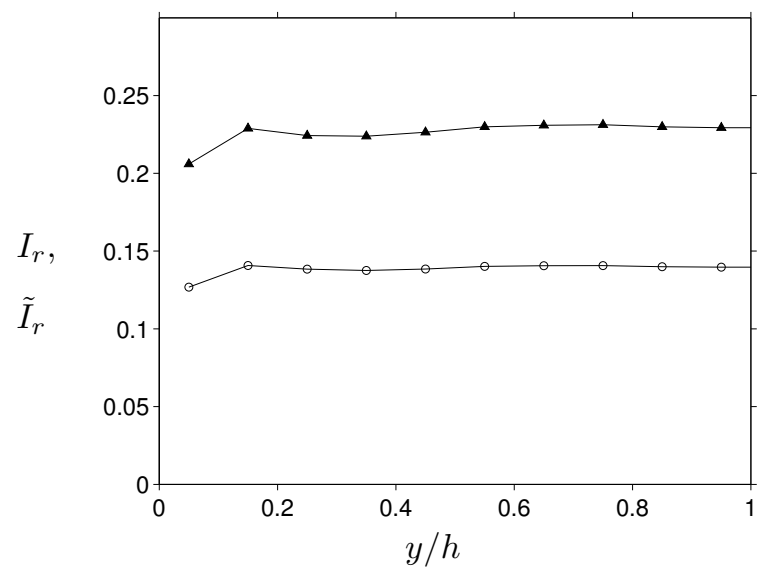

Figure 22: Relative turbulence intensity as a function of the wall-distance: $-\circ-, \tilde{I}_{r}=$ $\left(\left\langle u_{i}^{\prime} u_{i}^{\prime}\right\rangle / 3\right)^{1 / 2} / u_{\text {lag }} ;-, I_{r}=\left\langle u^{\prime} u^{\prime}\right\rangle^{1 / 2} / u_{\text {lag }}$;

\subsection{Particle-conditioned averaging}

In this section we turn our attention to the statistics of the flow in the neighborhood of the particles. For this purpose we have carried out averaging of the flow field conditioned upon the presence of particles. Before presenting a brief description of the literature on this topic (§ 3.4.1) as well as explaining the averaging procedure (§ 3.4.2), let us introduce the parameters which are believed to characterize the local flow field.

In addition to the Reynolds number based upon the average relative flow velocity and the sphere diameter $\left(R e_{\text {lag }}\right)$, the most prominent parameter describing turbulent flow around spheres is the relative turbulence intensity, i.e. the ratio between the intensity of the incoming fluid flow fluctuations and the apparent slip velocity. Whereas in homogeneous flows the definition of a relative turbulence intensity $\tilde{I}_{r}$ is often based upon the three-component turbulence intensity, viz. $\tilde{I}_{r}=\left(\left\langle u_{i}^{\prime} u_{i}^{\prime}\right\rangle / 3\right)^{1 / 2} / u_{\text {lag }}$, in cases with unidirectional mean flow (in the $x$-coordinate direction) the following definition is commonly employed: $I_{r}=\left\langle u^{\prime} u^{\prime}\right\rangle^{1 / 2} / u_{\text {lag }}$. In order to facilitate a relation of our current results to previous and future work, wall-normal profiles of the relative turbulence intensity according to both definitions are shown in figure 22. These profiles are found to be practically uniform across the channel, with values of $\tilde{I}_{r} \approx 0.14$ and $I_{r} \approx 0.23$.

\subsubsection{Previous work}

A number of previous studies provide useful reference data for the following discussion of our present results. Bagchi and Balachandar (2004) have simulated the flow around a single fixed particle in an unbounded domain, swept by homogeneous-isotropic turbulence (at $R e_{\lambda}=164$ ) with a superposed uniform mean flow. They considered two values of relative turbulence intensity, $\tilde{I}_{r}=0.1$ and 0.25 , while varying the particle Reynolds number based upon the average relative flow from $R e_{\text {lag }}=58$ to 610 . Although structurally different, homogeneous-isotropic flow is still relevant to plane channel flow if the central region of the latter configuration is considered, where a far less anisotropic state than in the near-wall region can be found. Therefore, the data of Bagchi and Balachandar (2004) will be compared to our present results in the vicinity of the centerplane of the channel.

Amoura et al. (2010) have investigated the flow around a fixed sphere swept by higher intensity incident turbulence (which was approximately homogeneous-isotropic) with experimental techniques. They have considered particle Reynolds numbers based upon the average relative flow from $R e_{\text {lag }}=100$ to 1000 , while the relative turbulence intensity was varied from $I_{r}=0.26$ to 0.45. At the same time, the ratio of particle diameter to integral length scale of the incident 
(a) $\frac{\tilde{z}}{R}$

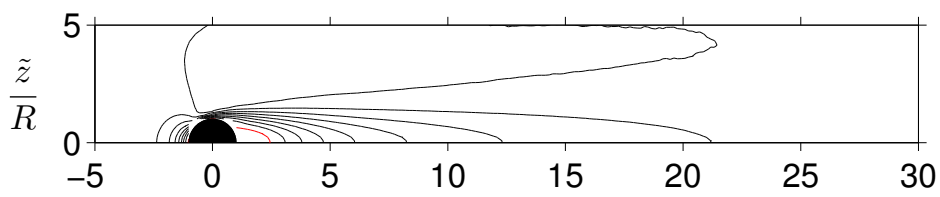

(b)

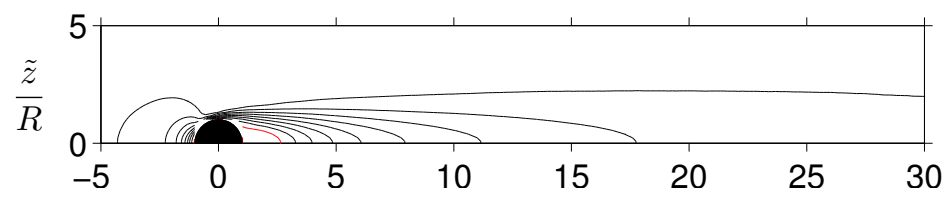

(c)

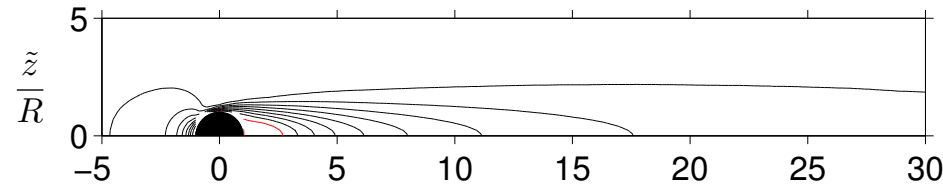

(d)

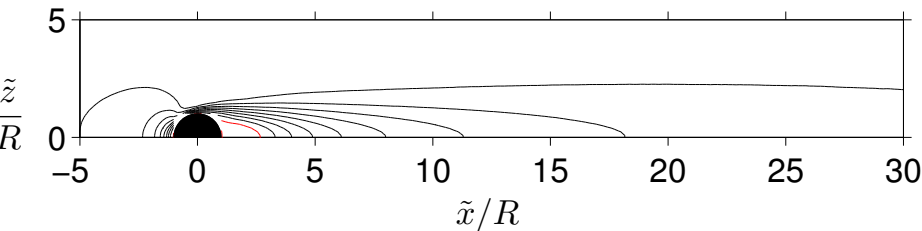

$y^{(s)} / h=0.05$,

$y^{(s)+}=11$,

$y^{(s)} / D=1$

$y^{(s)} / h=0.15$,

$y^{(s)+}=33$,

$y^{(s)} / D=3$

$y^{(s)} / h=0.35$,

$y^{(s)+}=77$,

$y^{(s)} / D=7$

$y^{(s)} / h=0.95$,

$y^{(s)+}=208$,

$y^{(s)} / D=19$

Figure 23: Contours of the streamwise component of the average relative velocity $\tilde{u}$ in wallparallel planes through the center of the particles, plotted for slabs at different wall-distances $y^{(s)}$. Contourlines are at uniformly distributed values $(0: .1: 0.9)$ times the maximum value in each plane. The zero-valued contour is plotted in red color.

turbulence was significantly larger than in most previous studies.

Wu and Faeth (1994) have performed experimental measurements of the flow around fixed spheres on the centerline of turbulent pipe flow $\left(R e_{b}=17000\right.$ up to 50000, based upon pipe radius). The particle Reynolds number based upon the average relative velocity was varied from $R e_{\text {lag }}=135$ to 1560 .

Legendre et al. (2006) have simulated the flow around a stationary particle on the centerline of turbulent pipe flow $\left(R e_{b}=3000\right.$ and $R e_{\tau}=200$, based upon the pipe radius) by means of LES. Their particle has a diameter of $D^{+}=10.23$ and a particle Reynolds number based upon the average relative velocity of $R e_{l a g}=200$, providing a parameter point which is not far removed from our present study. The prime difference is their relative turbulence intensity $\tilde{I}_{r}=0.037$, which is significantly lower than in the present case.

Zeng et al. (2010) have simulated turbulent channel flow $\left(R e_{\tau} \approx 180\right)$ with a single fixed particle, either located in the buffer layer $\left(y^{+} \approx 18\right)$ or at the center of the channel. They also varied the size of the particle in the range $D^{+}=3.5$ to 25 , such that a total of six different cases were considered. Out of that data-set, their case 2 is directly comparable to our data, featuring a particle with diameter $D^{+}=10.7$ placed in the buffer layer.

Please note that all of the studies mentioned above consider the flow around single particles which were fixed in space. Consequently, effects of particle mobility and collective effects were absent in those cases. We are not aware of previous work involving particle-conditioned averaging of turbulent flow-fields at the scale of the particles. One exception is the experimental work of Poelma, Westerweel and Ooms (2007) who did measure the conditionally-sampled flow field around mobile particles in decaying grid-generated turbulence. However, the near-field results presented in that reference are not sufficiently detailed in order to serve for the purpose of comparison in the present context. 


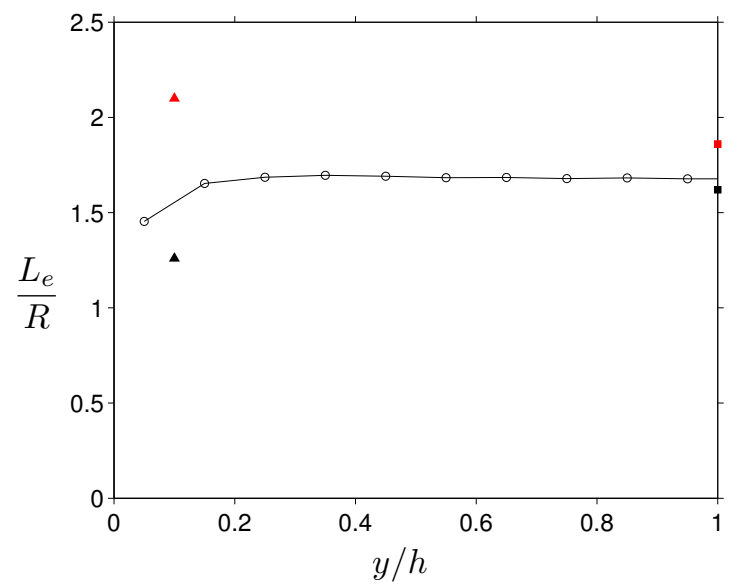

Figure 24: Average streamwise recirculation length $L_{e}$ in the wake of the particles, shown as a function of the wall-distance. Results for fixed single particles from the literature are indicated by the following symbols: $\boldsymbol{\Lambda}$, particle in buffer layer of turbulent channel flow (case 2 of Zeng et al., 2010); $\boldsymbol{\Delta}$, particle in buffer layer of laminar channel flow (Zeng et al., 2010); $\mathbf{\text { , particle }}$ in isotropic turbulence with mean relative-flow Reynolds number of $R e_{l a g}=114$ and relative turbulence intensity $\tilde{I}_{r}=0.25$ (case 4 of Bagchi and Balachandar, 2004); $\mathbf{n}$, particle in uniform flow at mean relative-flow Reynolds number of $\operatorname{Re}_{\text {lag }}=114$ (Bagchi and Balachandar, 2004).

\subsubsection{Preliminaries}

In the following we present an analysis of the local relative velocity field in the vicinity of the particles. For this purpose we have averaged the Eulerian velocity field in a frame of reference attached to the particle centers, summing over particles located in 20 wall-normal slabs (width of the slabs equal to $2 D$ ) as well as over a number of 97 instantaneous fields (yielding more than 70000 samples per slab). Before turning to the results, let us first make the averaging process more precise.

The instantaneous velocity difference between the two phases, computed with respect to a given particle, is defined as follows:

$$
\mathbf{u}_{r}^{(i)}(\tilde{\mathbf{x}}, t)=\mathbf{u}_{f}(\tilde{\mathbf{x}}, t)-\mathbf{u}_{p}^{(i)}(t)
$$

where the instantaneous coordinate relative to the $i$ th particle's center position $\mathbf{x}_{p}^{(i)}(t)$ is given by

$$
\tilde{\mathbf{x}}^{(i)}(t)=\mathbf{x}-\mathbf{x}_{p}^{(i)}(t) .
$$

Furthermore, in $(3) \mathbf{u}_{f}(\tilde{\mathbf{x}}, t)$ refers to the fluid velocity vector field and $\mathbf{u}_{p}^{(i)}(t)$ is the $i$ th particle's velocity vector at a given time $t$. The average relative velocity $\tilde{\mathbf{u}}\left(\tilde{\mathbf{x}}, y^{(s)}\right)$ in the slab centered at a wall-distance $y^{(s)}$ is computed from

$$
\tilde{\mathbf{u}}\left(\tilde{\mathbf{x}}, y^{(s)}\right)=\left\langle\mathbf{u}_{r}^{(i)}(\tilde{\mathbf{x}}, t)\right\rangle_{p, t},
$$

where the operator $\langle\cdot\rangle_{p, t}$ denotes averaging over all particles (whose center position is located in the respective slab) and over time (i.e. over a number of snapshots). Please note that averaging is performed over the actual volume instantaneously occupied by the fluid. This means that the volume occupied by other particles located in the neighborhood of a particular sphere is disregarded in the averaging procedure shown in (5). The reader is referred to Appendix A for a precise definition of the above averaging operator.

We can now decompose the instantaneous value of the relative velocity w.r.t. the $i$ th particle (3) into an average contribution (5) and a fluctuation $\mathbf{u}_{r}^{(i) \prime \prime}$, viz.

$$
\mathbf{u}_{r}^{(i)}(\tilde{\mathbf{x}}, t)=\tilde{\mathbf{u}}\left(\tilde{\mathbf{x}}, y^{(s)}\right)+\mathbf{u}_{r}^{(i) \prime \prime}(\tilde{\mathbf{x}}, t),
$$




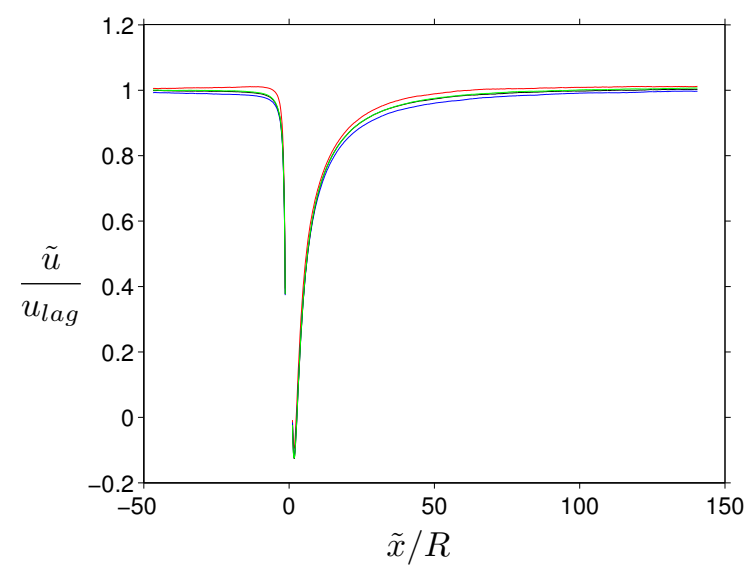

Figure 25: Average relative streamwise velocity $\tilde{u}$ along the streamwise axis through the center of the particles. The velocity $\tilde{u}$ is normalized by the apparent velocity lag $u_{\text {lag }}=\left|\left\langle u_{f}\right\rangle-\left\langle u_{p}\right\rangle\right|$. The solid curves correspond to particles being located at different wall-distances, averaged over bins centered at: $-y^{(s)+}=11,-y^{(s)+}=33,-y^{(s)+}=77,-y^{(s)+}=208$.

where the average of the fluctuation vanishes identically (i.e. $\left\langle\mathbf{u}_{r}^{(i) \prime \prime}\right\rangle_{p, t}=0$ ). From the fluctuations $\mathbf{u}_{r}^{(i) \prime \prime}(\mathbf{x}, t)$ implicitly defined in (6) we can compute second moments; for a component in the $\alpha$ direction the definition reads:

$$
\widetilde{u_{r, \alpha}^{\prime \prime} u_{r, \alpha}^{\prime \prime}}\left(\tilde{\mathbf{x}}, y^{(s)}\right)=\left\langle u_{r, \alpha}^{(i) \prime \prime}(\tilde{\mathbf{x}}, t) u_{r, \alpha}^{(i) \prime \prime}(\tilde{\mathbf{x}}, t)\right\rangle_{p, t},
$$

where no summation is implied for greek indices. Furthermore, we can define velocity fluctuations in the particle-centered frame of reference for each of the phases, viz.

$$
\begin{aligned}
\mathbf{u}_{f}^{(i) \prime \prime}(\tilde{\mathbf{x}}, t) & =\mathbf{u}_{f}(\tilde{\mathbf{x}}, t)-\left\langle\mathbf{u}_{f}(\tilde{\mathbf{x}}, t)\right\rangle_{p, t} \\
\mathbf{u}_{p}^{(i) \prime \prime}(t) & =\mathbf{u}_{p}^{(i)}(t)-\left\langle\mathbf{u}_{p}(t)\right\rangle_{p, t}
\end{aligned}
$$

such that $\mathbf{u}_{r}^{(i) \prime \prime}=\mathbf{u}_{f}^{(i) \prime \prime}-\mathbf{u}_{p}^{(i) \prime \prime}$.

\subsubsection{Mean relative velocity field}

Figure 23 shows the streamwise component of the average relative velocity, $\tilde{u}$, in wall-parallel planes through the particle center for averaging slabs at different wall-distances. It can be seen that the overall wake pattern in those planes is similar across the channel. However, the extent of the recirculation region as well as the deceleration (acceleration) upstream (downstream) of the particle are found to depend upon the wall distance. In particular, the region where the average streamwise velocity exhibits negative values is somewhat smaller at $y^{(s)+}=11$ than in the outer region. Likewise, the stagnation region upstream of the particle is more compact at this near-wall location $\left(y^{(s)+}=11\right)$ than at larger wall-distances. It can also be observed in figure 23 that there are no significant differences between the wake pattern of particles in the logarithmic layer and those located in the outer region or at the center of the channel.

The length of the recirculation region $L_{e}$, defined as the streamwise distance from the rear stagnation point to the downstream location where the average relative velocity changes sign, is shown in figure 24 as a function of wall-distance. Please note that $L_{e}$ is measured in the wallparallel plane passing through the particle center; therefore, the value of $L_{e}$ does not necessarily reflect the maximum length of the three-dimensional recirculation region, which might be located off-center. The figure shows an approximately constant value of $L_{e} / R \approx 1.72$ for wall-distances $y / h \geq 0.2\left(y^{+} \geq 45\right)$, where $R=D / 2$ is the particle radius. For smaller wall-distances, the length of the recirculation zone decreases, down to a value of $L_{e} / R=1.48$ in the first slab at $y^{(s)} / h=0.05\left(y^{(s)+}=11\right)$. 


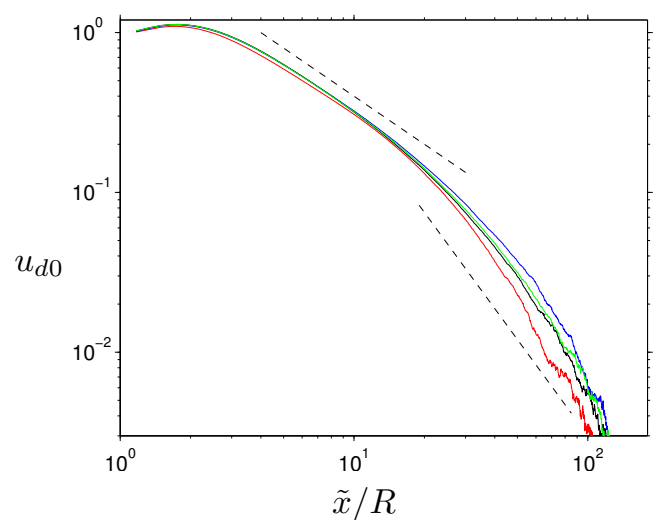

Figure 26: The streamwise velocity deficit $u_{d 0}$ along the streamwise axis through the center of the particles (on their downstream side). Color coding as in figure 25. The dashed straight lines indicate decay rates proportional to $x^{-1}$ and $x^{-2}$.

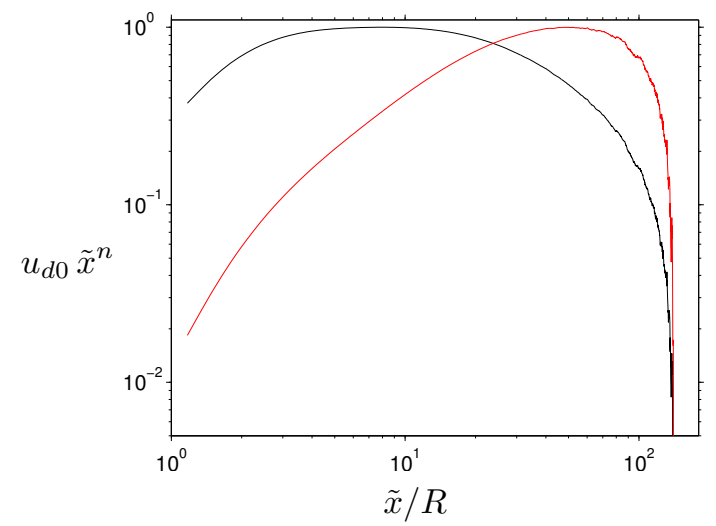

Figure 27: As in figure 26, but the velocity deficit is averaged over all particles located at $y / h \geq 0.2(y / D \geq 4)$, and the result is compensated in order to highlight powerlaw regions, i.e. the quantity $u_{d 0} \tilde{x}^{n}$ is plotted, where:,$- n=1 ;-, n=2$.

Figure 24 also includes data points for single fixed spheres in laminar channel and uniform flow (i.e. the laminar results reported by Bagchi and Balachandar, 2004; Zeng et al., 2010) at comparable average-flow particle Reynolds numbers. Since homogeneous-isotropic inflow was considered by Bagchi and Balachandar (2004), we relate their data to our results at the channel center. It can be seen that the presently obtained recirculation lengths fall below the reference values in laminar flow. This result is consistent with previous observations made with fixed spheres swept by turbulent flow: both Bagchi and Balachandar (2004) as well as Zeng et al. (2010) observed a reduction of the recirculation length due to background turbulence. Compared to the values of those authors (cf. black symbols in figure 24) our present results show somewhat larger recirculation lengths in turbulent background flow at comparable turbulence intensity. In particular, the present recirculation length in the buffer layer is approximately $26 \%$ higher than the value reported for case 2 of Zeng et al. (2010); the present results in the core of the channel are $6 \%$ higher than case 4 of Bagchi and Balachandar (2004). Although the differences are not extremely large, they might reflect the difference in the physics between our case and the single fixed particle configurations. Apart from possible effects of particle mobility, it should be remembered that the present flow field is significantly altered by the presence of particles, i.e. particle wakes are prominent features. Consequently, particles experience a turbulent flow field which is structurally significantly different from both the canonical channel flow and homogeneous-isotropic turbulence swept over fixed particles (Bagchi and Balachandar, 2004; Zeng et al., 2010).

Figure 25 shows the average relative velocity on the streamwise axis through the particle center. It should be noted that the curves do not quite reach the particle surface due to the interpolation during particle-centered averaging, as explained in Appendix A.1. In figure 25 the relative velocity $\tilde{u}$ is normalized by the apparent lag $u_{\text {lag }}$. It can be seen that with increasing distance from the particle, the average relative velocity tends to unity for all wall-distances. It is also visible from figure 25 that for particles located in the slab centered at $y^{(s)+}=11$ the approach to unity appears slightly faster (both on the upstream and the downstream side) as compared to particles at larger wall distances.

In order to further investigate the recovery of the average velocity in the wake, we define the normalized average velocity deficit in the wall-parallel plane passing through the particle center, viz.

$$
u_{d}\left(\tilde{x}, y^{(s)}, \tilde{z}\right)=\frac{\tilde{u}_{\infty}\left(y^{(s)}\right)-\tilde{u}\left((\tilde{x}, 0, \tilde{z})^{T}, y^{(s)}\right)}{\tilde{u}_{\infty}\left(y^{(s)}\right)},
$$




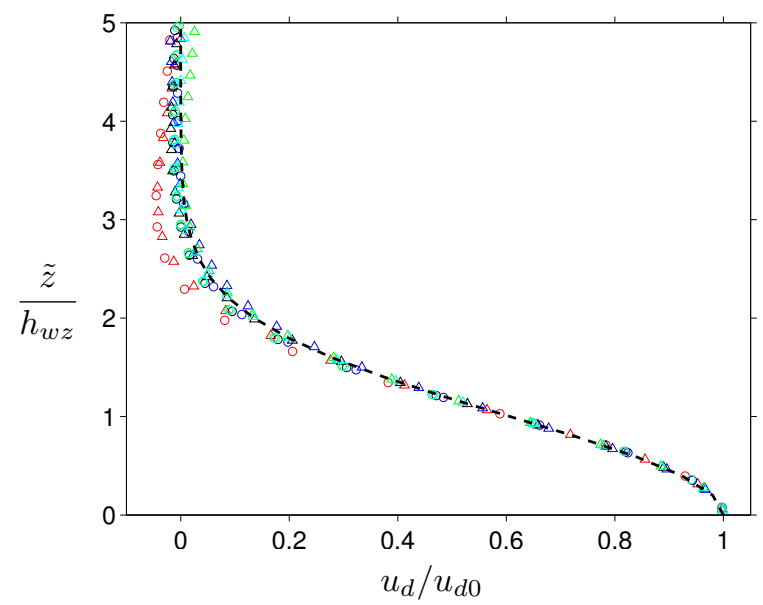

Figure 28: Average velocity deficit in the particle wakes as a function of the normalized spanwise coordinate, given at two downstream locations: $\circ, x / R=10 ; \Delta, x / R=20$. The color code indicates the wall-distance of the averaging slab (as given in figure 25). The dashed line corresponds to a Gaussian function as defined in (12).

where the average incoming relative velocity $\tilde{u}_{\infty}\left(y^{(s)}\right)$ is defined as the maximum (over $\tilde{x}$ ) of $\tilde{u}\left((\tilde{x}, 0,0)^{T}, y^{(s)}\right)$. The velocity deficit along the streamwise axis through the particle center, $u_{d 0}$, is then simply defined as follows

$$
u_{d 0}\left(\tilde{x}, y^{(s)}\right)=u_{d}\left(\tilde{x}, y^{(s)}, 0\right)
$$

Figure 26 shows the downstream evolution of the normalized velocity deficit $u_{d 0}$ in double logarithmic scale for distances of up to $140 R$. As observed in previous studies on turbulent flow around fixed single spheres (Wu and Faeth, 1994; Legendre et al., 2006; Amoura et al., 2010), roughly two regions can be distinguished: a near-wake zone with a decay rate approximately proportional to $x^{-1}$, and a far-wake with a decay of approximately $x^{-2}$. The velocity deficit for particles in the averaging slab adjacent to the wall $\left(y^{(s)+}=11\right)$ is somewhat smaller for distances up to $\tilde{x} / R \approx 10$, as already observed above. Otherwise, the curves corresponding to different wall-distances are equivalent to within statistical uncertainty. It has been observed by Legendre et al. (2006) that the change in slope (from $x^{-1}$ to $x^{-2}$ ) takes place at a downstream location where the velocity deficit and the turbulence intensity are of the same order $\left(u_{d 0} \approx\left\langle u^{\prime} u^{\prime}\right\rangle^{1 / 2}\right)$. The same is true in the present case: the change in slope occurs at approximately $\tilde{x} / R=25$, where the velocity deficit measures $u_{d 0} \approx 0.1$, which is indeed a value comparable to the turbulence intensity at the corresponding wall-normal locations (cf. figure $5 a$ ).

The extent of the regions where power-law behavior is observed can be deduced from figure 27 which shows the compensated velocity deficit $u_{d 0} \tilde{x}^{n}$ with exponents $n=1$ and $n=2$. In order to further increase the number of available samples, the data of all particles located at $y / h>0.2$ (i.e. slabs centered at $\left.y^{(s)} / h \geq 0.25\right)$ has been averaged. The figure shows that a decay of the velocity deficit in the particle wakes according to $x^{-2}$ takes place in a region approximately delimited by $40 \leq \tilde{x} / R \leq 80$. By way of comparison, in relatively low-intensity turbulence $\left(I_{r}=0.037\right)$ Legendre et al. (2006) found the $x^{-2}$ law to hold for distances above $x_{2}=50 R$, while Amoura et al. (2010) obtain the same evolution for $x_{2} \geq 5 R$ at higher turbulence intensity $\left(I_{r} \geq 0.26\right)$. The present results can therefore be qualified as consistent with the trend exhibited in these two studies.

Let us now turn to the question of self-similarity of the particle wakes. Wu and Faeth (1994) and subsequent authors (Bagchi and Balachandar, 2004; Legendre et al., 2006) observed that cross-stream profiles of the velocity deficit $u_{d}$ in the wake of isolated fixed spheres in turbulent 
(a)

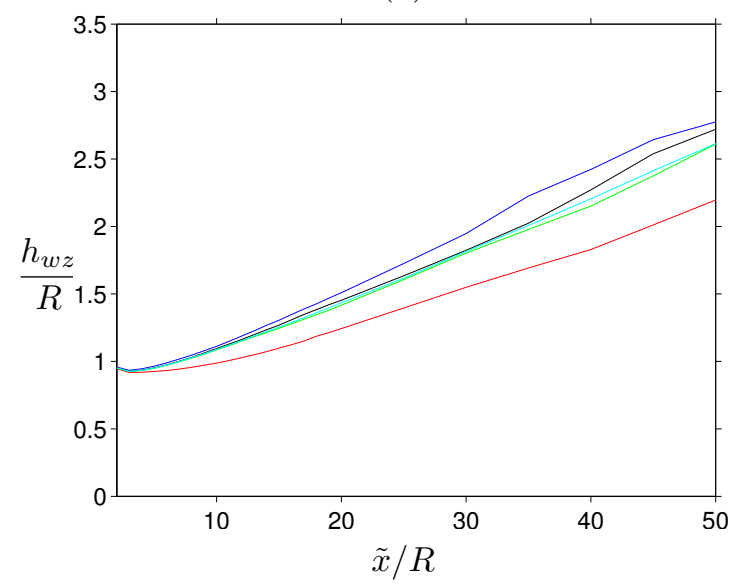

$(b)$

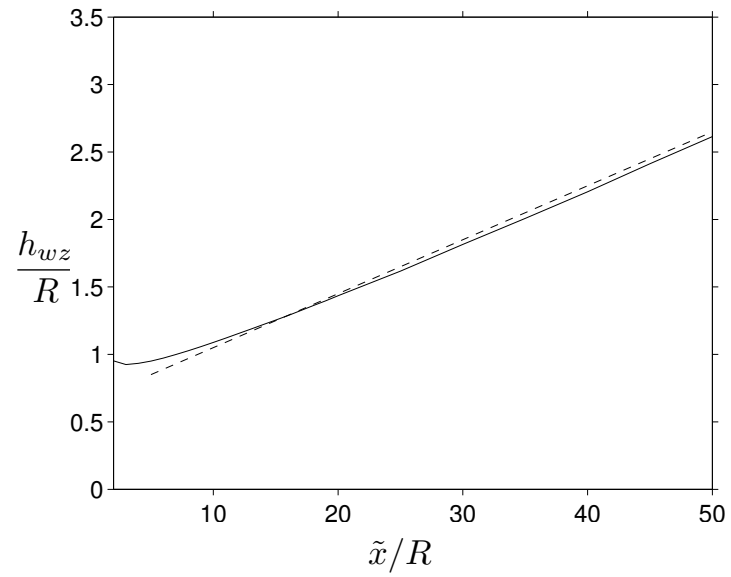

Figure 29: Spanwise half-width of the average particle wakes as a function of downstream distance. (a) shows data for individual wall-normal slabs, with color-coding as in figure 25. (b) shows data averaged over the core of the channel (for $y / h \geq 0.2$ ). The dashed line in $(b)$ is given by the function $h_{w z} / R=0.04 \tilde{x} / R+0.65$.

surroundings follow a Gaussian function, viz.

$$
\frac{u_{d}}{u_{d 0}}=\exp \left(-\frac{\tilde{z}^{2}}{2 h_{w z}^{2}}\right) .
$$

An appropriate length scale in (11) is the half-width $h_{w z}$ defined as the lateral position where the following relation holds:

$$
u_{d}\left(\tilde{z}=h_{w z}\right) / u_{d 0}=\exp (-1 / 2) .
$$

Figure 28 shows that the cross-wake profiles of the velocity deficit in the present case do indeed follow the Gaussian function (11) reasonably well; this is true for all wall-distances and over a substantial axial distance downstream of the particles. The streamwise evolution of the spanwise half-width $h_{w z}$ as defined in (12) is shown in figure 29. The graph in figure 29(a) demonstrates that the particles' wall-distance has a minor effect upon the wake half-width, as all curves have very similar evolutions; again only the averaging slab adjacent to the wall $\left(y^{(s)+}=11\right)$ represents a small exception, with a computed half-width which is systematically at slightly smaller values.

In figure $29(b)$ the core-averaged half-width (i.e. found upon averaging over all particles located at $y / h>0.2)$ is shown. A region of linear growth, i.e.

$$
h_{w z} / R=\alpha \tilde{x} / R+\beta,
$$

which extends over $15 \lesssim \tilde{x} / R \lesssim 50$ is observed. Let us recall that a linear expansion $h_{z w} \sim \tilde{x}$ is different from the case of spheres in uniform flow, in which laminar wakes obey $h_{z w} \sim \tilde{x}^{1 / 2}$ and turbulent wakes exhibit $h_{z w} \sim \tilde{x}^{1 / 3}$. The streamwise evolution in the present case (many mobile particles in turbulent background flow) is quite accurately represented by the coefficient values $\alpha=0.04$ and $\beta=0.65$ in the linear expansion law (13). At much lower relative turbulence intensity $\left(\tilde{I}_{r}=0.037\right)$, Legendre et al. (2006) have found a value of $\alpha=0.024$ for the wake expansion rate. Bagchi and Balachandar (2004), on the other hand, have obtained a value of $\alpha=0.135$ for their cases with relative turbulence intensity of $\tilde{I}_{r}=0.1$, independently of the particle Reynolds number. The analytic results of Eames et al. (2011) for wake spreading in turbulent surroundings do suggest a value of the expansion factor (in the linear spreading regime) comparable to the value of the relative turbulence intensity, viz. $\alpha \approx \tilde{I}_{r}$. This is apparently not the case in our present flow. However, it should be kept in mind that in the core of the channel, the dominant contribution of turbulent kinetic energy stems from the particle wakes themselves and not from 'incoming' 


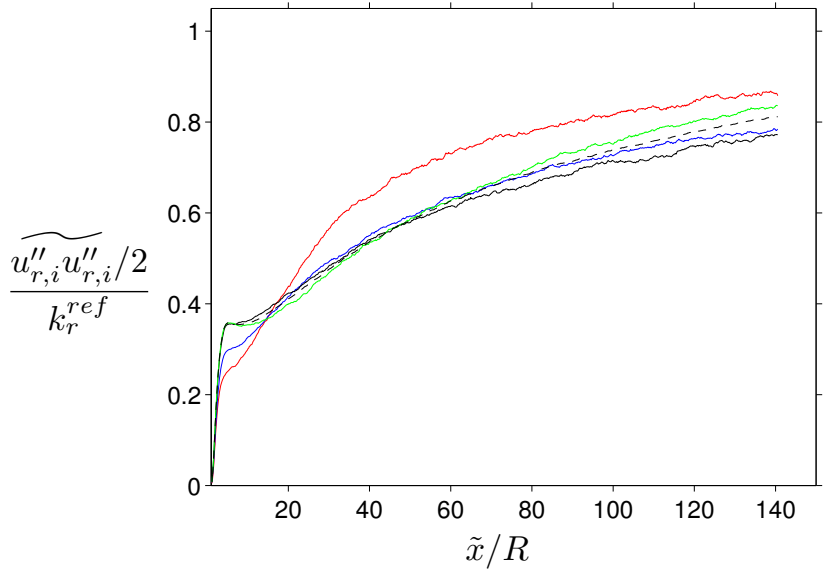

Figure 30: Energy of covariances between relative velocity fluctuations $\widetilde{u_{r, i}^{\prime \prime} u_{r, i}^{\prime \prime}}$ downstream of the particles, normalized by the reference kinetic energy given in (16) The color code indicates the wall-distance of the averaging slab (as given in figure 25). The dashed line corresponds to the data averaged over the core of the channel (for $y / h \geq 0.2$ ).

turbulence in the classical sense. Therefore, some of the assumptions made in the derivation of their model (in particular the homogeneous-isotropic structure of the turbulent flow field) do not appear to apply.

\subsubsection{Fluctuations}

By using the definition of the fluctuating velocities of each phase (8) the definition of the covariances between fluctuating relative velocities (7) can be rewritten as follows:

$$
\widehat{u_{r, \alpha}^{\prime \prime} u_{r, \alpha}^{\prime \prime}}\left(\tilde{\mathbf{x}}, y^{(s)}\right)=\left\langle u_{f, \alpha}^{\prime \prime}(\tilde{\mathbf{x}}, t) u_{f, \alpha}^{\prime \prime}(\tilde{\mathbf{x}}, t)\right\rangle_{p, t}+\left\langle u_{p, \alpha}^{(i) \prime \prime}(t) u_{p, \alpha}^{(i) \prime \prime}(t)\right\rangle_{p, t}-2\left\langle u_{f, \alpha}^{\prime \prime}(\tilde{\mathbf{x}}, t) u_{p, \alpha}^{(i) \prime \prime}(t)\right\rangle_{p, t} .
$$

This relation shows that the covariances of fluctuations w.r.t. the average defined in (5) result from three contributions: (i) covariances of the fluctuating fluid velocity field (conditioned on particle presence), (ii) covariances of particle velocity fluctuations, and (iii) covariances between particle velocity fluctuations and particle-conditioned fluid velocity fluctuations. For fixed particles the last two contributions in (14) vanish identically. Furthermore, for large distances from the particle (i.e. for large values of $\tilde{x}$ and/or $\tilde{z}$ ) we expect the last contribution (covariances between fluid and particle velocity fluctuations) to vanish. In that long-distance limit, the contribution from the particle-conditioned fluid velocity fluctuation covariances (first term on the r.h.s. of 14) is expected to tend towards the simple (unconditioned) plane-and-time-averaged fluid covariance $\left\langle u_{f, \alpha}^{\prime} u_{f, \alpha}^{\prime}\right\rangle$. Therefore, we define the following velocity scale:

$$
u_{r, \alpha}^{r e f}=\left(\left\langle u_{f, \alpha}^{\prime} u_{f, \alpha}^{\prime}\right\rangle+\left\langle u_{p, \alpha}^{(i) \prime \prime}(t) u_{p, \alpha}^{(i) \prime \prime}(t)\right\rangle_{p, t}\right)^{1 / 2}
$$

which is expected to allow for a reasonable normalization of the covariances of particle-conditioned relative velocities (7). From (15) we can derive a reference fluctuation energy $k_{r}^{r e f}$, viz.

$$
k_{r}^{r e f}=u_{r, i}^{r e f} u_{r, i}^{r e f} / 2 .
$$

The downstream evolution of fluctuation energy $\widetilde{u_{r, i}^{\prime \prime} u_{r, i}^{\prime \prime}} / 2$ on the axis through the particle center is shown in figure 30. At all wall-distances, the fluctuation energy increases from zero at the rear particle surface and tends towards unity at large downstream distances. For intermediate distances, two regimes can be distinguished. In the near-wake $(\tilde{x} / R \lesssim 10$, corresponding to the region where $u_{d 0} \sim \tilde{x}^{-1}$ ) a rapid increase of fluctuation energy is observed, which evolves 
approximately linearly with $\tilde{x}$. Further downstream, the approach towards unity is much slower, approximately following a logarithmic dependency on the downstream distance $\tilde{x}$.

\section{Conclusion}

In the present study we have revisited the case of vertical plane channel flow seeded with finite-size heavy particles already investigated by Uhlmann (2008). An additional DNS has been carried out with identical parameters as in the main simulation of that reference, except for the streamwise period of the computational domain which has been doubled.

The new dataset has first been compared to the previous simulation data of Uhlmann (2008), in order to determine the influence of the box size on the largest flow structures. It was observed that the columnar flow structures, which are induced by the presence of particles, are still not fully decorrelated in the prolonged domain. As a consequence, the Lagrangian auto-correlation of particle velocity is still biased by the fact that particles re-encounter long-lived flow structures after one return time (based upon the apparent slip velocity and the domain size). Results for average Eulerian quantities such as the mean fluid and particle velocity profiles are not strongly affected by the streamwise extension of the domain.

Furthermore, we have analyzed the new dataset with respect to additional aspects not previously considered in the context of vertical particulate channel flow. First, we have conducted an analysis of the spatial distribution of the disperse phase based upon Voronoi tessellation. It was found that the particles are less disorderly distributed than in a random case, slightly tending towards a homogeneous distribution. In addition, examining the aspect ratio of Voronoi cells has revealed that the particles exhibit a weak tendency to align in the streamwise direction, a trend which may be related to wake sheltering.

Second, we have carried out an analysis of the statistics related to particle acceleration. Near the wall, mean particle acceleration is found to significantly deviate from the mean acceleration of fluid particles. The standard deviation of particle acceleration, when normalized in wall units, differs among the three spatial components. When expressed as fluctuations of force coefficients (with approximate velocity scales based on the mean and rms fluid velocity of the corresponding components), this variability can be interpreted in terms of the standard drag law. Concerning the temporal correlation of particle acceleration data, we have observed a first zero-crossing after several viscous time units, followed by a slower decorrelation over several bulk time units. The p.d.f.'s of particle acceleration in the wall-normal and spanwise directions are found to be consistent with a lognormal distribution as proposed by Qureshi et al. (2008). The streamwise component, however, deviates from that lognormal fit, exhibiting significant positive skewness. One possible explanation for this positively-skewed acceleration p.d.f. is through a non-linear drag mechanism which would solely affect the streamwise component since it is the only spatial component with a finite apparent slip velocity. This point certainly merits further investigation.

Finally, we have performed particle-conditioned averaging of the flow field in the vicinity of the particles. It was found that the characteristics of the particle wakes in the present case are nearly independent of the wall distance, except for the near-wall region $(y / h \lesssim 0.2)$. The average length of the recirculation zone in the wake of the present particles (with relative flow Reynolds number based upon apparent slip velocity of 132 in the core of the channel) is consistent with data for single fixed spheres investigated by Bagchi and Balachandar (2004) and Zeng et al. (2010) at comparable Reynolds number and turbulence intensity. In particular, our results in the buffer layer (compared to case 2 of Zeng et al., 2010) and on the centerline (compared to case 4 of Bagchi and Balachandar, 2004) feature a somewhat larger mean wake length. The streamwise velocity deficit on the axis through the particles is found to decay as $x^{-1}$ in the near-wake and as $x^{-2}$ for distances beyond approximately 40 particle radii. As in previous studies involving fixed spheres in turbulent flow (Wu and Faeth, 1994; Bagchi and Balachandar, 2004; Legendre et al., 2006), the present average streamwise velocity profiles in the particles' wakes can be fit with reasonable agreement to an exponential function. The wake half-width resulting from the fit evolves almost linearly with the downstream distance over a considerable interval. The energy of 
the velocity fluctuations with respect to their particle-conditioned average, which is by definition zero on the particle surface, is found to evolve approximately linearly with downstream distance in the near-wake, and according to a logarithmic law in the far-wake.

\section{Acknowledgments}

The simulations were partially performed at the Barcelona Supercomputing Center, LRZ München and SCC Karlsruhe. The computer resources, technical expertise and assistance provided by these centers are thankfully acknowledged. AGK has received financial support through a FYS grant from KIT within the framework of the German Excellence Initiative. Thanks is also due to Véronique Roig, Frédéric Risso and Dominique Legendre for suggesting the analysis of particle wakes.

\section{References}

Amoura, Z., Roig, V., Risso, F., Billet, A.M., 2010. Attenuation of the wake of a sphere in an intense incident turbulence with large length scales. Phys. Fluids 22, 055105.

Bagchi, P., Balachandar, S., 2004. Response of the wake of an isolated particle to an isotropic turbulent flow. J. Fluid Mech. 518, 95-123.

Brown, R., Warhaft, Z., Voth, G., 2009. Acceleration statistics of neutrally buoyant spherical particles in intense turbulence. Phys. Rev. Lett. , 194501.

Calzavarini, E., Volk, R., Bourgoin, M., Lévéque, E., Pinton, J.F., Toschi, F., 2009. Acceleration statistics of finite-sized particles in turbulent flow: the role of faxén forces. J. Fluid Mech. 630, 179-189.

Caporaloni, M., Tampieri, F., Trombetti, F., Vittori, O., 1975. Transfer of particles in nonisotropic air turbulence. J. Atmosph. Sc. 32, 565-568.

Choi, J.I., Yeo, K., Lee, C., 2004. Lagrangian statistics in turbulent channel flow. Phys. Fluids 16, 779-793.

Clift, R., Grace, J., Weber, M., 1978. Bubbles, drops and particles. Academic Press.

Eames, I., Johnson, P.B., Roig, V., Risso, F., 2011. Effect of turbulence on the downstream velocity deficit of a rigid sphere. Phys. Fluids 23, 095103.

Ferenc, J.S., Neda, Z., 2007. On the size distribution of Poisson Voronoi cells. Physica A 385.

Fessler, J., Kulick, J., Eaton, J., 1994. Preferential concentration of heavy particles in a turbulent channel flow. Phys. Fluids 6, 3742-3749.

Fortes, A., Joseph, D., Lundgren, T., 1987. Nonlinear mechanics of fluidization of beds of spherical particles. J. Fluid Mech. 177, 467-483.

Gerashchenko, S., Sharp, N., Neuscamman, S., Warhaft, Z., 2008. Lagrangian measurements of inertial particle accelerations in a turbulent boundary layer. J. Fluid Mech. 617, 255-281.

Glowinski, R., Pan, T.W., Hesla, T., Joseph, D., 1999. A distributed Lagrange multiplier/fictitious domain method for particulate flows. Int. J. Multiphase Flow 25, 755-794.

Homann, H., Bec, J., 20010. Finite-size effects in the dynamics of neutrally buoyant particles in turbulent flow. J. Fluid Mech. 651, 81-91.

Kajishima, T., 2004. Numerical investigation of collective behaviour of gravitationally settling particles in a homogeneous field, in: Matsumoto, Y., Hishida, K., Tomiyama, A., Mishima, K., Hosokawa, S. (Eds.), Proc. ICMF 2004 (5th Int. Conf. Multiphase Flow), Yokohama.

Kajishima, T., Takiguchi, S., 2002. Interaction between particle clusters and particle-induced turbulence. Int. J. Heat Fluid Flow 23, 639-646.

Lavezzo, V., Soldati, A., Gerashchenko, S., Warhaft, Z., Collins, L.R., 2010. On the role of gravity and shear on inertial particle accelerations in near-wall turbulence. J. Fluid Mech. 658, 229-246.

Legendre, D., Merle, A., Magnaudet, J., 2006. Wake of a spherical bubble or a solid sphere set fixed in a turbulent environment. Phys. Fluids 18, 048102. 
Lucci, F., Ferrante, A., Elghobashi, S., 2010. Modulation of isotropic turbulence by particles of Taylor length-scale size. J. Fluid Mech. 650, 5-55.

Lucci, F., Ferrante, A., Elghobashi, S., 2011. Is Stokes number an appropriate indicator for turbulence modulation by particles of Taylor length-scale size. Phys. Fluids 23, 025101.

Melheim, J., 2005. Cluster integration method in Lagrangian particle dynamics. Comput. Phys. Commun. 171, 155-161.

Monchaux, R., Bourgoin, M., Cartellier, A., 2010. Preferential concentration of heavy particles: A Voronoï analysis. Phys. Fluids 22, 103304.

Monchaux, R., Bourgoin, M., Cartellier, A., 2012. Analyzing preferential concentration and clustering of inertial particles in turbulence. Int. J. Multiphase Flow 40, 1-18.

Mordant, N., Delour, J., Léveque, E., Arnéodo, A., Pinton, J., 2002. Long time correlations in Lagrangian dynamics: a key to intermittency in turbulence. Phys. Rev. Lett. 89, 254502.

Pan, Y., Banerjee, S., 1997. Numerical investigation of the effects of large particles on wallturbulence. Phys. Fluids 9, 3786-3807.

Parthasarathy, R., Faeth, G., 1990. Turbulence modulation in homogeneous dilute particle-laden flows. J. Fluid Mech. 220, 485-514.

Poelma, C., Westerweel, J., Ooms, G., 2007. Particle-fluid interactions in grid-generated turbulence. J. Fluid Mech. 589, 315-351.

Qureshi, N., Arrieta, U., Baudet, C., Cartellier, A., Gagne, Y., Bourgoin, M., 2008. Acceleration statistics of inertial particles in turbulent flow. Eur. Phys. J. B 66, 531-536.

Qureshi, N., Bourgoin, M., Baudet, C., Cartellier, A., Gagne, Y., 2007. Turbulent transport of material particles: an experimental study of finite-size effects. Phys. Rev. Lett. 99, 184502.

Reeks, M., 1983. The transport of discrete particles in inhomogeneous turbulence. J. Aerosol Sci. 14, 729-739.

Ten Cate, A., Derksen, J., Portella, L., Van Den Akker, H., 2004. Fully resolved simulations of colliding monodisperse spheres in forced isotropic turbulence. J. Fluid Mech. 519, 233-271.

Toschi, F., Bodenschatz, E., 2009. Lagrangian properties of particles in turbulence. Annu. Rev. Fluid Mech. 41, 375-404.

Uhlmann, M., 2005. An immersed boundary method with direct forcing for the simulation of particulate flows. J. Comput. Phys. 209, 448-476.

Uhlmann, M., 2008. Interface-resolved direct numerical simulation of vertical particulate channel flow in the turbulent regime. Phys. Fluids 20, 053305.

Warhaft, Z., 2009. Laboratory studies of droplets in turbulence: towards understanding the formation of clouds. Fluid Dyn. Res. 41, 011201.

Wu, J., Faeth, G., 1994. Sphere wakes at moderate Reynolds numbers in a turbulent environment. AIAA J. 32, 535-541.

Wu, J., Manasseh, R., 1998. Dynamics of dual-particles settling under gravity. Int. J. Multiphase Flow 24, 1343-1358.

Wylie, J., Koch, D., 2000. Particle clustering due to hydrodynamical interactions. Phys. Fluids 12, 964-970.

Xu, H., Bodenschatz, E., 2008. Motion of inertial particles with size larger than Kolmogorov scale in turbulent flows. Physica D 237, 2095-2100.

Yeo, K., Kim, B.G., Lee, C., 2010. On the near-wall characteristics of acceleration in turbulence. J. Fluid Mech. 659, 405-419.

Zeng, L., Balachandar, S., Najjar, F., 2010. Wake response of a stationary finite-sized particle in a turbulent channel flow. Int. J. Multiphase Flow 36, 406-422. 


\section{Appendix A Averaging procedures}

\section{A.1 Particle-centered averaging}

Let us define an indicator function $\phi_{b i n}^{(j)}(y)$ which signals whether a given wall-normal position $y$ is located inside or outside a particular wall-normal slab with index $j$, viz.

$$
\phi_{\text {bin }}^{(j)}(y)=\left\{\begin{array}{ll}
1 & \text { if } \quad(j-1) \frac{2 h}{N_{b i n}} \leq y<j \frac{2 h}{N_{b i n}} \\
0 & \text { else }
\end{array},\right.
$$

where $N_{b i n}$ is the number of slabs used to span the channel width. Similarly, we define an indicator function $\phi_{f}(\mathbf{x}, t)$ for the fluid phase:

$$
\phi_{f}(\mathbf{x}, t)=\left\{\begin{array}{ll}
1 & \text { if } \\
0 & \text { else }
\end{array} \quad \mathbf{x} \in \Omega_{f}(t),\right.
$$

where $\Omega_{f}(t)$ is the part of the computational domain $\Omega$ which is occupied by fluid at time $t$. We can now define a discrete counter field $\tilde{n}_{i j k}^{(s)}$ which holds the number of samples obtained through averaging at a given grid node with indices $i, j, k$ for a given $y$-slab with index $s$, viz.

$$
\tilde{n}_{i j k}^{(s)}=\sum_{m=1}^{N_{\text {snap }}} \sum_{l=1}^{N_{p}} \phi_{b i n}^{(s)}\left(y_{p}^{(l)}\left(t^{m}\right)\right) \phi_{f}\left(\tilde{\mathbf{x}}_{i j k}^{(l)}\left(t^{m}\right), t^{m}\right)
$$

In equation (19) the symbol $t^{m}$ indicates the time corresponding to the $m$ th snapshot in the database (comprising a total of $N_{\text {snap }}$ snapshots) and $\tilde{\mathbf{x}}_{i j k}^{(l)}\left(t^{m}\right)$ is a coordinate relative to the $l$ th particle's center position at time $t^{m}$, as defined in (4).

The actual averaging can now be performed analogously to (19), including a division by the local number of samples. For a vector field $\boldsymbol{\xi}(\mathbf{x}, t)$ we define the averaging operator as follows:

$$
\langle\boldsymbol{\xi}\rangle_{p, t}\left(\tilde{\mathbf{x}}_{i j k}, y^{(s)}\right)=\frac{1}{\tilde{n}_{i j k}^{(s)}} \sum_{m=1}^{N_{\text {snap }}} \sum_{l=1}^{N_{p}} \phi_{b i n}^{(s)}\left(y_{p}^{(l)}\left(t^{m}\right)\right) \phi_{f}\left(\tilde{\mathbf{x}}_{i j k}^{(l)}\left(t^{m}\right), t^{m}\right) \boldsymbol{\xi}\left(\tilde{\mathbf{x}}_{i j k}^{(l)}\left(t^{m}\right), t^{m}\right) .
$$

In practice the coordinates $\tilde{\mathbf{x}}_{i j k}^{(l)}\left(t^{m}\right)$ of the grid nodes covering the predefined averaging volume do not coincide with the grid used in the direct numerical simulation. This means that the vector field to be averaged ( $\boldsymbol{\xi}$ in equation 20 ) is not directly available at the coordinates $\tilde{\mathbf{x}}_{i j k}^{(l)}\left(t^{m}\right)$. Therefore, the averaging process defined in (20) involves spatial interpolation, which has been realized with a tri-linear formula. As a consequence of the use of the fluid indicator function, the particle-centered average fields do not quite reach the particle surface.

The number of (uniformly spaced) bins for evaluating averages defined by (20) was chosen as $N_{\text {bin }}=20$. The number of snapshots amounts to $N_{\text {snap }}=97$, yielding approximately 80000 samples per slab. Further averaging over all bins with $y^{(s)} / h \geq 0.25$ (also referred to as "coreaveraging" in the main text) yields approximately 650000 samples for the quantities shown in figures 27 and $29(b)$.

\section{A.2 Wall-parallel plane and time averaging}

The standard averaging over wall-parallel planes and time (not conditioned upon particle presence) can now conveniently be defined using the notation and the indicator functions already introduced in A.1. Let us first define a counter of fluid sample points in a wall-parallel plane at a given walldistance with index $j$, viz.

$$
n_{j}=\sum_{m=1}^{N_{t i m e}} \sum_{i=1}^{N_{x}} \sum_{k=1}^{N_{z}} \phi_{f}\left(\mathbf{x}_{i j k}, t^{m}\right) .
$$

Note that the number of data-sets $N_{\text {time }}$ used for this quantity is much larger than $N_{\text {snap }}$ in the case of particle-centered averaging (equations 19 and 20), since standard averaging is performed 
(a)

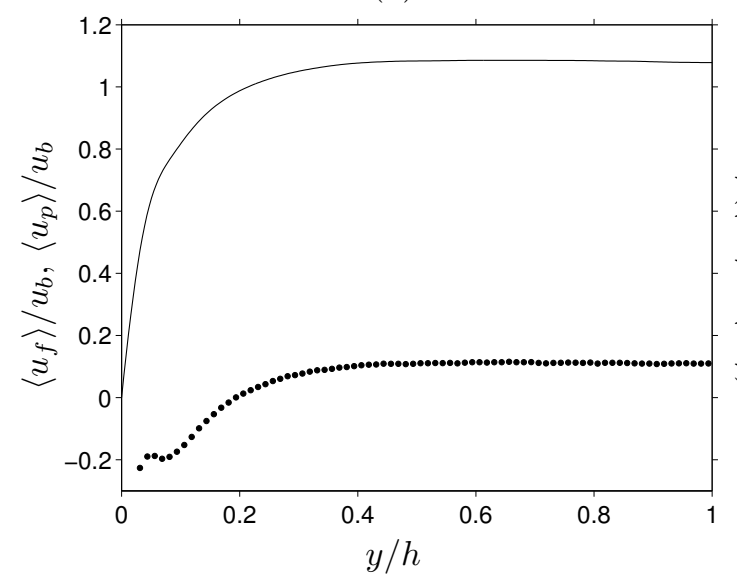

(b)

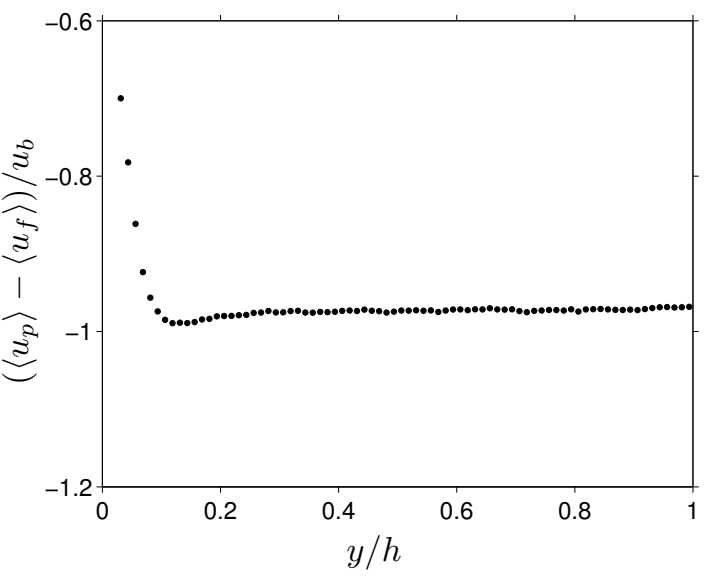

Figure 31: (a) Mean velocity profiles of both phases in the present simulation: ——- fluid phase; -, solid phase. (b) Apparent slip velocity between the phases. The fluid phase data is averaged according to the operator defined in (22).

at runtime, while the latter is carried out at a post-processing stage. Averaging over wall-parallel planes and in time, while considering only grid points being located in the fluid domain, is defined as follows:

$$
\langle\boldsymbol{\xi}\rangle\left(y_{j}\right)=\frac{1}{n_{j}} \sum_{m=1}^{N_{\text {time }}} \sum_{i=1}^{N_{x}} \sum_{k=1}^{N_{z}} \phi_{f}\left(\mathbf{x}_{i j k}, t^{m}\right) \boldsymbol{\xi}\left(\mathbf{x}_{i j k}, t^{m}\right) .
$$

Consequently, $\langle\boldsymbol{\xi}\rangle$ is a function of wall-distance alone, whereas $\langle\boldsymbol{\xi}\rangle_{p, t}$ is a three-dimensional field for each wall-normal slab $y^{(s)}$.

Note that purely for the purpose of strict comparison between the present simulation results and data from Uhlmann (2008), the following averaging operator which does not distinguishes between the solid and fluid phases is used ('composite' averaging, cf. figures 3 and 5):

$$
\langle\boldsymbol{\xi}\rangle_{c}\left(y_{j}\right)=\frac{1}{N_{\text {time }} N_{x} N_{z}} \sum_{m=1}^{N_{\text {time }}} \sum_{i=1}^{N_{x}} \sum_{k=1}^{N_{z}} \boldsymbol{\xi}\left(\mathbf{x}_{i j k}, t^{m}\right) .
$$

\section{A.3 Binned averages over particle-related quantities}

Concerning Lagrangian quantities, we employ averages over wall-normal bins, using the indicator function given in (17). The sample counter for each bin with index $s$ is computed over the number $N_{\text {time }}^{(p)}$ of available particle fields and summing over all particles, viz.

$$
\hat{n}^{(s)}=\sum_{m=1}^{N_{\text {time }}^{(p)}} \sum_{l=1}^{N_{p}} \phi_{b i n}^{(s)}\left(y_{p}^{(l)}\left(t^{m}\right)\right) .
$$

The binned average (over time and the number of particles) of a Lagrangian quantity $\zeta_{p}$ is defined as follows:

$$
\left\langle\boldsymbol{\zeta}_{\boldsymbol{p}}\right\rangle\left(y^{(s)}\right)=\frac{1}{\hat{n}^{(s)}} \sum_{m=1}^{N_{\text {time }}^{(p)}} \sum_{l=1}^{N_{p}} \phi_{\text {bin }}^{(s)}\left(y_{p}^{(l)}\left(t^{m}\right)\right) \boldsymbol{\zeta}_{\boldsymbol{p}}{ }^{(l)}\left(t^{m}\right) .
$$

In this manuscript we have chosen the number of (uniformly spaced) bins for evaluating averages defined by (25) as $N_{b i n}=160$. The present data corresponds to a total number of approximately $1.8 \cdot 10^{7}$ samples. 
$(a)$

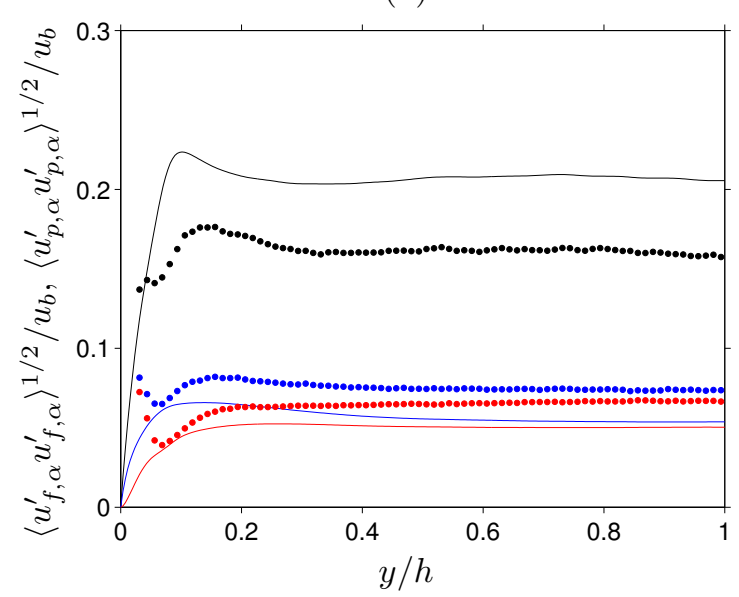

(b)

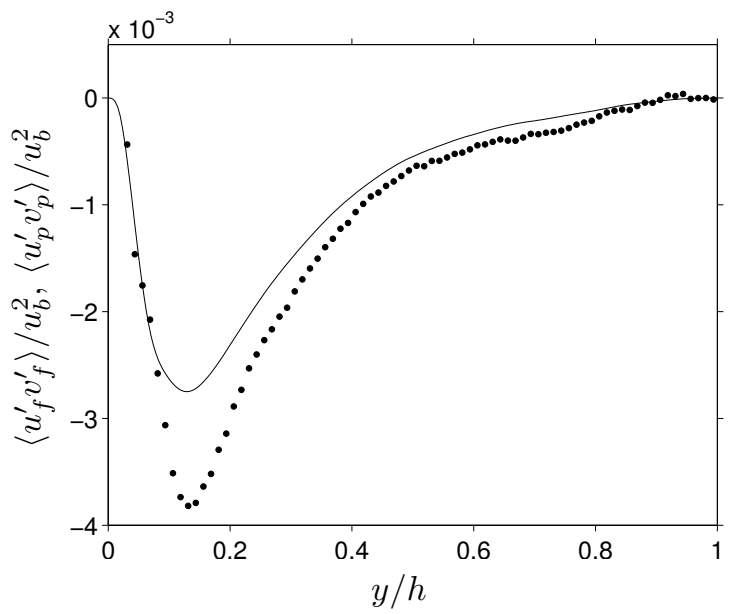

Figure 32: (a) R.m.s. of velocity fluctuations of both phases in the present simulation, with lines corresponding to the fluid phase, and symbols to the particulate phase. The color coding indicates streamwise (black), wall-normal (red), spanwise (blue) components. (b) Reynolds shear stress of fluid velocity fluctuations as well as corresponding velocity correlation of the particle motion. All quantities are normalized in bulk units. The fluid phase data is averaged according to the operator defined in (22).

\section{Appendix B Eulerian statistics for the present simulation}

Figures 31 and 32 show the same quantities as presented in figures 3 and 5 (for the present simulation), but defined with the correct averaging operator which only considers grid nodes being instantaneously located in the fluid domain (cf. definition in equation 22). These quantities are presented for future reference. 